\title{
1 A computational method for predicting the most likely 2 evolutionary trajectories in the stepwise accumulation of 3 resistance mutations
}

4 R. Charlotte Eccleston ${ }^{\mathrm{a}, 1}$, Emilia Manko ${ }^{\mathrm{a}}$, Susana Campino ${ }^{\mathrm{a}}$, Taane G. Clarke ${ }^{\mathrm{a}, \mathrm{b}}$, Nicholas

5 Furnham $^{\mathrm{a}}$

6 aDepartment of Infection Biology, London School of Hygiene and Tropical Medicine, London, 7 United Kingdom; 'bepartment of Infectious Disease Epidemiology, London School of Hygiene

8 and Tropical Medicine, London, United Kingdom

$9 \quad{ }^{1}$ R. Charlotte Eccleston

10 Email: charlotte.eccleston@Ishtm.ac.uk

11 Author Contributions: R.C.E., S.C., T.G.C. and N.F. conceived and designed the study. E.M. performed the bioinformatic processing of the raw sequencing data. R.C.E performed the research. R.C.E. analyzed the data. R.C.E. wrote the first draft of paper. All authors commented on and edited the manuscript and approved the final version. R.C.E. compiled the final

15 manuscript.

16 Competing Interest Statement: None.

17 Classification: Major: Biological Sciences, Minor: Biophysics and Computational Biology, 18 Evolution

19 Keywords: Antimicrobial resistance, epistasis, pathogen evolution

\section{This PDF file includes:}

Main Text

Figures 1 to 8

Tables 1 to 3 


\section{Abstract}

32 Pathogen evolution of drug resistance often occurs in a stepwise manner via accumulation of

33 multiple mutations, which in combination have a non-additive impact on fitness, a phenomenon

34 known as epistasis. Epistasis complicates the sequence-structure-function relationship and

35 undermines our ability to predict evolution. We present a computational method to predict

36 evolutionary trajectories that accounts for epistasis, using the Rosetta Flex ddG protocol to

37 estimate drug binding free energy changes upon mutation and an evolutionary model based in

38 thermodynamics and statistical mechanics. We apply this method to predict evolutionary

39 trajectories to known multiple mutations associated with resistant phenotypes in malaria.

40 Resistance to the combination drug sulfadoxine-pyrimethamine (SP) in malaria-causing species

41 Plasmodium falciparum (Pf) and Plasmodium vivax ( $P v)$ has arisen via the accumulation of

42 multiple point mutations in the DHFR and DHPS genes. Four known PfDHFR pyrimethamine

43 resistance mutations are highly prevalent in field-isolates and multiple studies have shown

44 epistatic interactions between these mutations determine the accessible evolutionary trajectories

45 to the highly resistant quadruple mutation N51I,C59R,S108N,I164L. We simulated the possible

46 evolutionary trajectories to this quadruple PfDHFR mutation as well as the homologous PVDHFR

47 mutations. In both cases, our most probable pathways agreed well with those determined

48 experimentally. We also applied this method to predict the most likely evolutionary pathways to

49 observed multiple mutations associated with sulfadoxine resistance in PfDHPS and PVDHPS.

50 This novel method can be applied to any drug-target system where the drug acts by binding to

51 the target. 


\section{Significance Statement}

55 Antimicrobial resistance $(\mathrm{AMR})$ is a major public health threat, resulting from the overuse and

56 misuse of antimicrobial drugs. Our ability to monitor emerging resistance and direct the most

57 appropriate treatment strategy (stewardship) would be strengthened by the development of

58 methods to accurately predict the mutational pathways that lead to resistance. We present a

59 computational method to predict the most likely resistance pathways that result from mutations

60 that alter drug binding affinity. We utilized the Rosetta Flex ddG protocol and a thermodynamic

61 evolutionary model. This novel approach can accurately capture known resistance trajectories

62 and can be applied to any system where resistance arises via changes in drug binding affinity.

\section{Main Text}

\section{Introduction}

65 The development of new antimicrobial drugs and therapeutics, and the design of more successful

66 drug deployment strategies to reduce the prevalence of resistance, requires an understanding of

67 the underlying molecular evolution.

68 Antimicrobial resistance (AMR) poses a huge global health threat by a wide range of mechanisms

69 including: the protection of drug targets by post-translational modifications such as methylation;

70 the presence and increased expression of proteins that bind to targets preventing drug

71 interactions; limiting the physical entry of the drug compound to the cell or increasing the

72 efficiency of drug efflux through the type or number of efflux pumps, or in the case of bacteria,

73 horizontal gene transfer [1-3]. One of the major routes to resistance, and a focus of the work

74 presented here, is genomic variation within protein coding regions. Of particular significance are

75 single-nucleotide polymorphisms (SNPs) in the antimicrobial target gene that alter the protein

76 structure and prevent efficient binding of the antimicrobial drug. Provided these SNPs do not

77 prevent the target from carrying out its function, the resistant strains will proliferate within the

78 population [4]. 
79 The evolution of resistance alleles is affected by the evolutionary interplay between selection for

80 resistance and selection for protein function, as well as drug concentration, epistasis between

81 resistant mutations and mutational bias [5-8]. Prime examples of this are the Plasmodium

82 falciparum and vivax parasites that cause the majority of malaria infections and which have

83 evolved strong resistance to many antimalarial drugs, including pyrimethamine [9], chloroquinine

$84 \quad[10]$ and sulfadoxine [11].

85 There were an estimated 229 million new cases of malaria world-wide in 2019, resulting in

86 approximately 409,000 deaths predominately among children under 5 [12]. P. falciparum

87 accounts for the majority of cases in Africa, Southeast Asia, the Eastern Mediterranean and

88 Western Pacific, whilst P. vivax is predominant in Central and South America.

89 P. falciparum malaria has been treated with the combination drug sulfadoxine-pyrimethamine

90 (SP) since 1970s, which targets the folate metabolic pathway. Numerous resistance mutations

91 have arisen within its genome as a result of SNPs in $P$. falciparum dihydrofolate reductase

92 (PfDHFR) and dihydropteroate synthase (PfDHPS) genes, which are the targets of

93 pyrimethamine and sulfadoxine respectively $[11,13,14]$. Although SP is not usually used to treat

$94 P$. vivax, co-infections with $P$. falciparum have meant SP resistance mutations have also arisen in

95 the P. vivax genome [15]. The enzymes of the folate pathway are largely conserved across

96 Plasmodium species, and so polymorphisms in equivalent positions have been observed in $P$.

97 vivax DHFR (PVDHFR) and DHPS (PVDHPS) and are thought to confer resistance to SP [16-19]

98 The DHFR gene encodes an enzyme that uses NADPH to synthesize tetrahydrofolate, a co-

99 factor in the synthesis of amino acids [20], and pyrimethamine acts to disrupt this process,

100 thereby blocking DNA synthesis and slowing down growth. Stepwise acquisition of multiple

101 mutations leading to resistance to pyrimethamine has been observed in both PfDHFR [7,9] and

102 PVDHFR [8]. DHPS exists as a bifunctional enzyme with hydroxymethyldihydropterin

103 pyrophosphokinase (HPPK) and performs a key step in folate synthesis by catalysing the

104 conversion of para-aminobenzoate (pABA) to dihydropteroate. The anti-folate drug sulfadoxine 
105 targets DHPS by preventing this conversion and mutations in DHPS have arisen that confer

106 resistance to sulfadoxine [21, 22].

107 In PfDHFR, a combination of four mutations - Asn-51 to lle (N51I), Cys-59 to Arg (C59R), Ser-

108108 to Asn (S108N) and lle-164 to Leu (I164L) - has been reported to result in resistance to

109 pyrimethamine [23] by altering the binding pocket and reducing the affinity for the drug [24]. Non-

110 additivity in both pyrimethamine binding free energy and the concentration required to inhibit cell

111 growth by $50 \%$ (IC50) has been observed experimentally for combinations of these four

112 mutations [7, 9]. This non-additivity - known as epistasis - means that mutations which on their

113 own are not associated with a resistance phenotype, could do so when in combination with other

114 mutations. Epistasis between these mutations has been shown to determine the evolutionary

115 trajectories to the quadruple mutation N51I,C59R,S108N,I164L, which is strongly associated with

116 pyrimethamine resistance.

117 A similar investigation was conducted into the homologous set of PVDHFR mutations - Asn-50 to

118 Ile (N50I), Ser-59 to Arg (S58R), Ser-117 to Asn (S117N) and Ile-173 to Leu (I173L) - and the

119 accessible evolutionary trajectories to the quadruple mutation [8], some combinations of which

120 have been observed to result in pyrimethamine resistance both in vivo and in vitro [19, 25].

121 Evolutionary simulations accounting for growth rates, IC50 measurements for increasing

122 concentrations of pyrimethamine and nucleotide bias predicted the most likely pathways to the

123 quadruple mutation for different drug concentrations. They observed the trajectories at each

124 concentration were influenced by epistasis between the mutations and the adaptive conflict

125 between endogenous function and acquisition of drug resistance.

126 These studies, along with other investigations [5, 26] have highlighted the prevalence of epistasis

127 among resistance mutations and the importance of considering epistatic interactions between

128 mutations when predicting evolutionary trajectories of drug resistance.

129 Epistasis between mutations within the same protein arises due to energetic interactions between 130 the amino acids, where the impact of a mutation on either function, fitness or a physical property 
131 depends upon the protein sequence [27]. When epistasis occurs between two or more mutations,

132 their combined impact on protein fitness or a physical trait such as stability or binding affinity,

133 does not equal the sum of their independent impacts. As well as the aforementioned studies,

134 epistasis been observed in the evolution of many pathogens, such as Escherichia coli [28],

135 Influenza [29], and RNA viruses [30]. Epistasis may also have important consequences for the

136 success of AMR management strategies that aim to reduce resistance via the cessation of use of

137 a particular drug. In theory, stopping the use of a drug should result in reversion of resistance

138 mutations, because most resistance mutations incur a large fitness cost in the absence of drug

139 [31-33]. However, the success of this strategy has been mixed, and in some cases bacterial

140 populations remained resistant [34-36], which may be the result of compensatory mutations (a

141 type of epistasis), which mitigate the deleterious impact of resistance mutations, allowing them to

142 remain in a population and thus retain resistance even in the absence of drug selection pressures

143 [37].

144 Therefore, understanding how epistasis arises and predicting which mutations will interact

145 epistatically, is important for anticipating future mutations, designing new drugs and developing

146 strategies to minimize resistance and predicting the most likely evolutionary trajectories to

147 resistance.

148 Whilst experimental methods have been successful in characterising the interactions between

149 resistance mutations and predicting evolutionary trajectories, they are expensive and time

150 consuming. Therefore, identifying computational methods that can accurately characterise how

151 mutations interact could potentially enable fast and reliable predictions of evolutionary trajectories

152 to resistance.

153 One of the main mechanisms of drug resistance is selection for mutations that reduce drug

154 binding free energy. Several computational tools exist to predict changes in binding free energy

155 upon mutation, however their ability to predict epistasis is limited or unknown. The Rosetta

156 protocol Flex ddG [38] is the current state-of-the art method for predicting changes in protein- 
157 protein and protein-ligand binding free energy. Rosetta is a software suite for macromolecular

158 modelling and design that uses all-atom mixed physics- and knowledge-based potentials, and

159 provides a diverse set of protocols to perform specific tasks, such as structure prediction,

160 molecular docking and homology modelling [39]. The Flex ddG protocol has been found to

161 perform better than machine learning methods and comparably to molecular dynamics methods

162 when tested on a large dataset of ligand binding free energy changes upon protein mutation [40,

163 41]. However, its ability to capture epistasis has not yet been tested. Therefore, we investigated

164 how well Flex ddG can capture epistasis between mutations and observed a good correlation with

165 experimental data.

166 Next, we developed a method for predicting evolutionary trajectories to multiple resistance

167 mutations, using predicted binding free energy changes and an evolutionary model based in

168 thermodynamics and statistical mechanics. We tested this method by simulating the accessible

169 evolutionary trajectories to known resistant quadruple mutants in both PfDHFR and PVDHFR, as

170 these targets have been studied experimentally. We observed good agreement between the most

171 likely trajectories predicted by our model and those predicted experimentally.

172 Once we had demonstrated our method for predicting evolutionary trajectories agrees well with

173 experimentally determined trajectories, we extended our analysis to the DHPS gene of $P$.

174 falciparum and P.vivax. This work represents the first attempt to predict evolutionary trajectories

175 to resistance in these genes, either experimentally or computationally.

176 We compared our predictions to the frequency of mutations found in isolate data from countries

177 from several geographical regions including South America, West and East Africa, South and

178 Southeast Asia and Melanesia. 


\section{Results}

180 Rosetta Flex-ddG accurately predicts binding free energy changes and epistasis for PfDHFR 181 mutations

182 We investigated if Flex ddG, was able to capture experimentally measured non-additivity in 183 binding free energy. The change in binding free energy was predicted for the combinatorically 184 complete set of the four PfDHFR pyrimethamine resistance mutations N511, C59R, S108N and 185 I164L. The authors of the Flex ddG protocol suggest conducting a minimum of 35 runs and taking 186 the average of the distribution as the prediction for that mutation. We observed that the 187 distributions were not well characterised by only 35 runs and so we undertook 150 runs for each 188 mutation. Similarly, many of the distributions were not Normal and so we chose to use the peak of 189 the distribution as a prediction for each mutation.

190 We compared our predictions with the data of Sirawaraporn et al. (1997) [9] who experimentally 191 determined the change in binding free energy for a subset of the combinatorically complete set of 192 the four known PfDHFR resistance mutations (Table 1), where a positive $\Delta G$ value indicates a 193 destabilising mutation and a negative $\Delta G$ value indicates a stabilising mutation (Note: Rosetta

194 Flex ddG calculates the change in binding free energy as $\Delta \Delta G=\Delta G_{m u t}-\Delta G_{W T}$, whereas

195 Sirawaraporn et al. (1997) calculated the change as the reverse, $\Delta \Delta G=\Delta G_{W T}-\Delta G_{m u t}$, where 196 WT indicates the wild-type free energy and mut indicates the mutant free energy. Therefore, in 197 the original manuscript, a mutation that destabilised the binding corresponded to a negative $\Delta \Delta G$, 198 whilst here we have reversed the signs of the original data to enable comparison with our 199 predictions. Furthermore, Flex ddG predictions are in Rosetta Energy Units (R.E.U), whereas 200 data from [9] is in units $\mathrm{kcal} / \mathrm{mol}$ ).

201 Our predictions had a Pearson's correlation of 0.63 with the experimental dataset and 8/9 of the 202 mutations were correctly classified as either stabilising or destabilising. These metrics show that 203 the predictions capture the experimental data satisfactorily. The single mutation S108N was 204 found to be the only destabilising single mutation in both the experimental data and the 
205 predictions, with all other single mutations having a stabilising impact on pyrimethamine binding.

206 There are, however, some discrepancies, namely in the experimental data the double mutation

$207 \mathrm{~N} 51 \mathrm{l} / \mathrm{S} 108 \mathrm{~N}$ is more destabilizing to binding than single mutation $\mathrm{S} 108 \mathrm{~N}$, however the peak of the

208 Flex ddG distribution was stabilizing, with only a few of the 150 runs predicting the mutation was

209 destabilizing. The triple mutation C59R/S108N/I164L was found experimentally to be the most

210 destabilizing of the triple mutations, however Flex ddG predicted it to be only mildly destabilizing

211 and the least destabilizing of the triple mutations. Furthermore, the quadruple mutation was found

212 experimentally to have the most destabilising impact out of all combinations of single and multiple

213 mutations, however, Flex ddG predicted it to be less destabilising than the double mutation

214 C59R/S108N.

215 Sirawaraporn et al. (1997) determined the 'interaction energy', which they defined as the

216 difference between the change in binding free energy due to a multiple mutation and the sum of

217 the changes in binding free energy when the mutations occur on their own. For a double

218 mutation, the interaction energy is the same as the pairwise epistasis between the pair of

219 mutations. For triple and quadruple mutations, the interaction energy quantifies the non-additivity

220 between the mutations and indicates the level of epistasis between the mutations.

221 We determined the interaction energy by finding the difference between the predicted change in

222 binding free energy of a multiple mutation and the sum of the predictions of their independent

223 binding free energy changes (Table 1). A positive value of the interaction energy indicates the

224 sum of the independent impacts is more destabilizing than the impact of the multiple mutation and

225 a negative value indicates the sum is less destabilizing than the combined impact.

226 A correlation of 0.765 with the data was observed and $4 / 5$ values were correctly classified as

227 having either a positive or negative interaction energy. The incorrectly classified mutation was

228 again N511/S108N, where the interaction energy was predicted to be positive, but found

229 experimentally to be negative, because the sum of the individual predictions was destabilising but

230 the double mutation itself was predicted to have a stabilising impact. 
231 Both the experimental data and our predictions found that the quadruple mutation had the largest

232 magnitude interaction energy reflecting the greatest difference between the stabilising impact of

233 the sum of the individual mutations and the destabilising impact of the quadruple mutation itself.

234 The triple mutation C59R,S108N,I164L was found to have a large negative interaction energy in

235 both the data and our predictions, where the triple mutation was found to be destabilizing whilst

236 the sum of the individual impacts was stabilising to pyrimethamine binding. We also observed

237 large negative interaction energy between S108N and C59R, where C59R is stabilizing in the

238 wildtype background but destabilizing in the background of $\mathrm{S} 108 \mathrm{~N}$, an example of sign epistasis

239 and in agreement with the observations of both Sirawaraporn et al. (1997) and Lozovosky et al.

240 (2009).

241 However, whilst the interaction energy of the triple mutation N51I,C59R,S108N was negative for

242 both data and predictions, in our predictions its magnitude was much smaller compared to the

243 data. Both single mutations N51I and C59R were predicted to be only marginally stabilising -

244 almost neutral - to pyrimethamine binding, whilst in the experimental data both mutations have a

245 large stabilising impact. Furthermore, the triple mutation was predicted to be only marginally more

246 destabilising than single mutation $\mathrm{S} 108 \mathrm{~N}$, resulting in the small negative interaction energy.

\section{A thermodynamic evolutionary model can predict the most likely evolutionary}

248 trajectories to quadruple mutations in both PfDHFR and PvDHFR

249 We simulated the evolutionary trajectories to quadruple mutant N51I,C59R,S108N,I164L, using

250 an evolutionary model, adapted from previous studies [42-44], in which selection acts to reduce

251 the binding affinity between PfDHFR and pyrimethamine. Briefly, starting from the wild-type

252 protein, we randomly sample a value from the Flex ddG distributions for each of the four single

253 mutations and calculate the fitness of the mutated protein (Eq. 1), and the fixation probability (Eq.

254 2). A mutation is then chosen with a probability proportional to the fixation probability and this is 
255 repeated until the quadruple mutation is reached. We carried out 10,000 runs and determined the

256 probability of each trajectory.

257 The top two most likely trajectories were S108N/C59R/N51I/I164L, S108N/C59R/I164L/N51I,

258 respectively (Figure 1a), corresponding to the top two most likely pathways determined in [7]. The

259 fourth most likely pathway predicted by our model, S108N/N51I/C59R/164L, corresponds to the

260 third most likely pathway predicted by [7].

261 We compared our predictions to the frequency of mutations observed in our isolate data (Figure

262 1b). In the isolate data, S108N was the most frequent single mutation, C59R,S108N the most

263 frequent double mutation and N51I,C59R,S108N the most frequent triple mutation. This suggests

264 the pathway proceeds in the order S108N/C59R/N51I/I164L, in agreement with the most likely

265 pathway from both our evolutionary simulations and those using experimental data.

266 Single mutations C59R and N51I are the second and third most prevalent single mutations,

267 respectively, in both our isolate data and our pathway predictions and I164L is absent from the

268 isolate data and has a zero probability of being selected as the first step of our evolutionary

269 trajectories. Whilst there is agreement between the model and the data regarding the most

270 common double mutation (C59R,S108N), the agreement is less convincing for the remaining

271 double mutations. For example, N51I,S108N is the second most frequent double mutation in the

272 isolate data but the fourth most likely double mutation predicted by the model when summing up

273 all pathways. There is better agreement with the triple mutations, where C59R,S108N,I164L is

274 the second most frequent triple in the data and the second most likely triple in the simulations

275 when summing over all possible pathways.

276 We also compared our pathway predictions to the distributions of mutations from isolates from

277 different geographical areas. We grouped the isolate data in to five geographical regions: South

278 America (Brazil, Colombia and Peru), West Africa (Benin, Burkina Faso, Cameroon, Cape Verde,

279 Cote d'Ivoire, Gabon, Gambia, Ghana, Guinea, Mali, Mauritania, Nigeria and Senegal), Central

280 and East Africa (Congo, Eritrea, Ethiopia, Kenya, Madagascar, Malawi, Tanzania, Uganda), 
281 Southeast Asia (Bangladesh, Cambodia, Indonesia, Laos, Myanmar, Thailand and Vietnam) and

282 Melanesia (Papua New Guinea).

284 Examining the geographical distribution of PfDHFR mutations (Figure 2), we can see that some

285 combinations of the aforementioned set of four resistance mutations are observed in high

286 frequencies in most regions (See Supplementary data 'PfDHFR_pcnt.csv'). By considering the

287 frequencies of the possible combinations of the four mutations N51I, C59R, S108N and I164L, we

288 can infer the likely evolutionary pathways to pyrimethamine resistance followed in each region

289 and compare with our predicted pathways.

290

291 The evolution of pyrimethamine resistance in West Africa and Southeast Asia appears to have

292 followed the two main pathways predicted here and in previous studies, S108N/C59R/N51I/164L

293 and/or S108N/C59R/I164L/N51I.

294 In West Africa, the most frequent mutations were single mutation S108N, double mutation

295 C59R,S108N and triple mutation N51I,C59R,S108N, which were found in $1.2 \%, 8.1 \%, 67.0 \%$ of

296 isolates respectively. The quadruple mutation N51I,C59R,S108N,I164L, single mutations C59R,

297 and N51I and double mutations N51I,C59R and N51I,S108N were each found in $>1 \%$ of isolates.

298 This suggests West Africa is following the main trajectory S108N/C59R/N511/164L.

299 In Southeast Asia, single mutation S108N, double mutation C59R,S108N, triple mutation

$300 \mathrm{~N} 51$ I,C59R,S108N and quadruple mutation N51I,C59R,S108N,I164L were observed in >1\%,

$3018.2 \%, 34.2 \%$ and $52.5 \%$ of isolates, respectively. Additionally, double mutants N51I,C59R,

$302 \mathrm{~N} 51 \mathrm{I}, \mathrm{S} 108 \mathrm{~N}$ and S108N,I164L were each observed in $>1 \%$ of isolates and triple mutants

$303 \mathrm{~N} 511, \mathrm{C} 59 \mathrm{R}, \mathrm{I164L}$ and C59R,S108N,I164L were observed in $>1 \%$ and $3.7 \%$ of isolates,

304 respectively. This suggests the two most likely pathways to pyrimethamine resistance in this

305 region are S108N/C59R/N511//164L and S108N/C59R/I164L/N51I. 
307 However, not all regions follow the most likely trajectories predicted by our evolutionary model.

308 The evolution of pyrimethamine resistance in Central and East Africa appears to have followed a

309 slightly different pathway. The three most common mutations were double mutations N51I,S108N

310 and C59R,S108N and triple mutation N51I,C59R,S108N which were found in $12.1 \%, 2.9 \%$ and

$31182.3 \%$ of isolates respectively. Single mutation S108N, double mutation N51I,C59R, triple

312 mutation N51I,S108N,I164L and quadruple mutation N51I,C59R,S108N,I164L were each found in

$313>1 \%$ of isolates. All other combinations of the four mutations were absent. This suggests the main

314 trajectory in this region is $\mathrm{S} 108 \mathrm{~N} / \mathrm{N} 51 \mathrm{l} / \mathrm{C} 59 \mathrm{R} / \mathrm{l} 164 \mathrm{~L}$, in addition to a second less likely trajectory

315 S108N/C59R/N51I/I164L.

316 The distribution of mutations in South America also suggests alternative routes to pyrimethamine

317 resistance. The most frequent mutations were single mutation S108N, double mutation

$318 \mathrm{~N} 51 \mathrm{I}, \mathrm{S} 108 \mathrm{~N}$ and triple mutation N51I,S108N,I164L which accounted for $52.0 \%, 24.0 \%$ and $8.0 \%$

319 of the isolates, respectively. All other combinations of the four mutations were not observed in

320 South America. This suggests this region is following the trajectory S108N/N51I/I164L to the triple

321 mutation.

322 In Papua New Guinea, however, out of all possible combinations of the four mutations, only

323 single mutation $\mathrm{S} 108 \mathrm{~N}$ and double mutation C59R,S108N were observed in $>1 \%$ and $19.3 \%$ of

324 isolates, respectively. Triple mutation C59R,S108N,S306F was observed in the remaining 79.8\%

325 of isolates.

326 From the geographical distribution we can see that there are a small number of other mutations

327 that appear at non-negligible frequencies. In South America, the single mutation A16V was

328 observed in $2.0 \%$ of isolates and the triple mutation C50R,N51I,S108N is observed in $8.0 \%$ of

329 isolates.

331 Next, we generated Flex ddG predictions of the change in binding free energy for each mutation

332 in the combinatorically complete set of four mutations in PVDHFR, (N50I, S58R, S117N and 
333 1173L), which are homologous to the four in P. falciparum. We used these predictions to simulate

334 the evolutionary trajectories to the quadruple mutation (Figure 3a) and compared our results to

335 those presented in Jiang et al. (2013) [8]. In [8] they consider the wild-type allele to have a Serine

336 at codons 58 and 117 and therefore we do the same for ease of comparison. The most likely first

337 step in our model is S58R, which Flex ddG predicts to be the only single mutation to reduce the

338 binding affinity when considering both the average and the peak of the distribution (Figure S1).

339 Single mutation $\mathrm{S} 117 \mathrm{~N}$ reduces the binding affinity on average, but the peak of the distribution is

340 a mild stabilizing effect (Figure S2).

341 The most likely pathway predicted by our simulations (S58R/S117N/I173L/N50I) corresponds to

342 the second most likely pathway predicted by Jiang et al. (2013) [8] for the highest pyrimethamine

343 concentration. Our second most likely pathway (S58R/S117N/N50I/1173L) corresponds to the first

344 most likely pathway predicted in [8] for the highest pyrimethamine concentration.

345 We compared the frequency of the 16 possible combinations of mutations in the pathway to the

346 frequency found in our P. vivax isolate data (Figure 3b). Mutations S117N and S58R were the top

347 two most frequent single mutations in our isolate data, respectively. The double mutant

348 S58R,S117N was the most frequent mutation of the set found in our isolate data and was the

349 most likely double mutation in our simulations when considering all possible routes to each of the

350 six possible double mutants. The only triple mutant observed in the data was S58R,S117N,I173L

351 and this was the most likely of the four possible triple mutations in our simulations when

352 considering all possible routes. The quadruple mutation N50I,S58R,S117N,I173L is not observed

353 in our isolate data, and has not been observed in the literature either.

354 We grouped the data into five broad geographical regions: South America (Brazil, Colombia,

355 Guyana, Panama, Peru), East Africa (Ethiopia, Eritrea, Madagascar, Sudan, Uganda), South Asia

356 (Afghanistan, Bangladesh, India, Pakistan, Sri Lanka), Southeast Asia (Cambodia, China, Laos,

357 Malaysia, Myanmar, Philippines, Thailand and Vietnam) and Melanesia (Papua New Guinea). 
358 If we consider the geographical distribution of mutations in PVDHFR (Figure 4), we can see the

359 dominant mutations differs between regions and compared to PfDHFR (Figure 2) there is a more

360 diverse set of mutations that occur at non-negligible frequencies. As mentioned previously, the

361 quadruple mutation is not observed in the isolate data and so we will infer trajectories up to triple

362 mutant combinations of the four mutations N50I, S58R, S117N, I173L.

363 In South America, single mutations S117N and S58R and double mutation S58R,S117N were

364 observed in $13.2 \%, 3.5 \%$ and $28.8 \%$ of isolates respectively. Double mutation $\mathrm{S} 117 \mathrm{~N}, \mathrm{I} 173 \mathrm{~L}$ and

365 triple mutation S58R,S117N,I173L were both observed in $>1 \%$ of isolates. This suggests the

366 evolution to triple mutation S58R,S117N,I173L follows a main trajectory S117N/S58R/1173L and

367 two less likely alternative trajectories S58R/S117N/I173L and S117N/1173L/S58R.

368 However, in East Africa, South Asia, Southeast Asia and Papua New Guinea, only S117N, S58R

369 and S58R,S117N are observed out of the possible mutational combinations.

371 In addition to the set of four mutations studied here, we observed other PVDHFR mutations in our

372 isolate data. The double mutation $\mathrm{S} 58 \mathrm{R}, \mathrm{S} 117 \mathrm{~N}$ is now so frequent in the population that both

373 mutations are considered fixed and the allele containing the substitutions at those residues is

374 considered the major allele [45]. Therefore, we also observe mutations N117T and R58K at low

375 frequencies in our dataset as these are mutations that occur following the Asparagine substitution

376 at position 117 and the Arginine substitution at position 58.

377

378 Two quadruple mutations F57L,S58R,T61M,N117T and F57I,S58R,T61M,N117T were also found

379 in high frequency in our isolate data. Mutation F57L,S58R,T61M,N117T was found in 38.5\% of

380 isolates from Papua New Guinea and $15.1 \%$ of isolates from Southeast Asia, whilst

381 F57I,S58R,T61M,N117T was found in $29.7 \%$ of isolates from Southeast Asia. Intermediates of

382 these two quadruple mutations were also found in our isolate data. The high frequency of these 
quadruple mutations suggests it may result in a resistant phenotype and future work could study

384 the trajectories to these mutations.

Predicting the most likely evolutionary pathways to multiple resistant mutations in PfDHPS

The PfDHPS mutations Ser-436 to Ala (S436A), Ala-437 to Gly (A437G), Lys-540 to Glu (K540E) and Ala-581 to Gly (A581G) are highly prevalent in isolate data and have been shown to confer resistance to sulfadoxine, both independently and in combination. In our data, the quadruple mutation S436A,A437G,K540E,A581G is observed, along with high frequency intermediate

392 single, double and triple mutant combinations.

We generated Flex ddG predictions of the change in binding free energy upon mutation for the combinatorically complete set of these four mutations. Triglia et al. (1997) [46] reported a 10-fold decrease in sulfadoxine binding affinity for single mutation A437G, but only a 4-fold decrease in binding affinity for A581G. Double mutations A437G/A581G and S436A/A437G were reported to result in a further 10 -fold decrease in binding affinity, with a 70 -fold decrease for A437G/K540E, relative to $A 437 G$ alone. These results suggest these mutations are interacting epistatically.

We compared the peak of our predicted distributions for each mutation to the data presented in

400 Triglia et al. (1997) [46] (Table 2). We observed Pearson's correlation of 0.469 with 5/5 correctly

401 classified as having a destabilizing impact. In the data, single mutation A437G was found to have

402 a greater destabilizing impact than A581G. If we consider the average of the distributions, A437G

403 was found to be around 50 times more destabilizing than A581G $\left(<\Delta \Delta G_{A 437 G}>=0.259,<\right.$

$404 \Delta \Delta G_{A 581 G}>=0.005$ (R.E.U)). However, if we consider the peak of the distributions, A437G has a

405 smaller destabilizing impact than A581G $\left(\Delta \Delta G_{A 437 G}^{*}=0.025, \Delta \Delta G_{A 581 G}^{*}=0.11\right.$ (R.E.U)). If we

406 analyze the overall distribution of A437G (Fig. S3) we can see that whilst the peak of the

407 distribution occurs around small destabilizing changes in free energy and there is a long tail

408 where many of the runs have predicted a much larger destabilizing impact, with a maximum of 
4091.58 (R.E.U), and only 2/150 runs predicted a stabilizing impact. The A581G distribution (Fig. S4),

410 however, has a maximum destabilizing prediction of just 0.246 , with almost half $(65 / 150)$ of the

411 runs predicting a stabilizing effect. This again highlights the need to consider the entire

412 distribution of predictions for a specific mutation, instead of a summarizing statistic, when

413 predicting the most likely evolutionary pathways.

414 We simulated the possible evolutionary trajectories to known PfDHPS sulfadoxine quadruple 415 mutation S436A,A437G,K540E,A581G (Figure 5a). The most likely first step was single mutation 416 A437G, in agreement with previous studies which found A437G was the most frequent single

417 mutation sulfadoxine-resistant PfDHPS isolates [13, 47] and the data presented in [46].

418 Furthermore, A437G is now considered fixed in the population [48] and A437G is also the most

419 common mutation in our isolate data, followed by S436A (Figure 5b). The most likely double

420 mutation in our simulations was A437G/S436A, followed by A437G/K540E. This corresponds well

421 to our isolate data in which the two most likely double mutations were A437G,K540E and

422 S436A,A437G, respectively. The most likely triple mutation from our simulations was

$423 \mathrm{~A} 437 \mathrm{G} / \mathrm{K} 540 \mathrm{E} / \mathrm{A} 581 \mathrm{G}$ followed by A437G/A581G/K540E and the two most frequent triple

424 mutations in our isolate data were A437G,K540E,A581G and S436A,A437G,K540E, respectively.

425 The most likely pathway to the quadruple mutation was A437G/S436A/A581G/K540E, followed

426 by $\mathrm{A} 437 \mathrm{G} / \mathrm{K} 540 \mathrm{E} / \mathrm{A} 581 \mathrm{G} / \mathrm{S} 436 \mathrm{~A}$. In our isolate data, the quadruple mutation is not very common

427 and is only found in $1 / 6038$ isolates.

428 We grouped the isolate data in to five geographical regions: South America (Brazil, Colombia and 429 Peru), West Africa (Benin, Burkina Faso, Cameroon, Cape Verde, Cote d'Ivoire, Gabon, Gambia, 430 Ghana, Guinea, Mali, Mauritania, Nigeria and Senegal), East Africa (Congo, Eritrea, Ethiopia,

431 Kenya, Madagascar, Malawi, Tanzania, Uganda), Southeast Asia (Bangladesh, Cambodia,

432 Indonesia, Laos, Myanmar, Thailand and Vietnam) and Melanesia (Papua New Guinea).

433 In Southeast Asia, the most common mutations were single mutation A437G, double mutations 434 S436A,A437G, A437G,K540E and A437G,A581G and triple mutations S436A,A437G,K540E and 
435 A437G,K540E,A581G which were observed in $4.6 \%, 5.8 \%, 6 \%, 2.3 \%, 21.5 \%$ and $28.6 \%$ of

436 isolates, respectively. Single mutations S436A and K540E were observed in $1.1 \%$ and $>1 \%$ of

437 isolates, respectively and the quadruple mutation S436A,A437G,K540E,A581G was observed in

$438>1 \%$ of isolates. This suggests three possible trajectories to the quadruple mutation with

439 A437G/K540E/A581G/S436A being the most likely pathway, followed by

$440 \mathrm{~A} 437 \mathrm{G} / \mathrm{K} 540 \mathrm{E} / \mathrm{S} 436 \mathrm{~A} / \mathrm{A} 581 \mathrm{G}$ and $\mathrm{A} 437 \mathrm{G} / \mathrm{S} 436 \mathrm{~A} / \mathrm{K} 540 \mathrm{E} / \mathrm{A} 581 \mathrm{G}$ as the second and third most

441 likely pathways, respectively.

442 In South America, the most common mutations were single mutation A437G and double mutation

443 A437G,A581G which were both found in $10.0 \%$ of isolates and triple mutation

$444 \mathrm{~A} 437 \mathrm{G}, \mathrm{K} 540 \mathrm{E}, \mathrm{A} 581 \mathrm{G}$ which was observed in $16.0 \%$ of isolates. All other combinations of the four 445 mutations were not observed in the South America isolates. This suggests the pathway followed

446 to the triple mutation is $\mathrm{A} 437 \mathrm{G} / \mathrm{A} 581 \mathrm{G} / \mathrm{K} 540 \mathrm{E}$.

447 In West Africa, single mutations A437G and S436A were observed in $41.9 \%$ and $17.2 \%$ of

448 isolates, respectively, double mutations S436A,A437G and A437G,K540E were observed in

$44918.0 \%$ and $>1 \%$ of isolates, respectively and triple mutation A437G,K540E,A581G was observed

450 in $>1 \%$ of isolates. This suggests separate evolutionary trajectories to double mutation

451 S436A,A437G and triple mutation A437G,K540E,A581G. The main trajectory to the

452 S436G,A437G is A437G/S436A with a second slightly less likely trajectory S436A/A437G, whilst

453 the trajectory to the triple mutation likely occurs A437G/K540E/A581G.

454 In East Africa, the most common mutations were single mutation A437G, double mutation

455 A437G,K540E and triple mutation A437G,K540E,A581G which were observed in $24.8 \%, 57 \%$

456 and $7.3 \%$ of isolates, respectively. Single mutations S436A and K540E were observed in 1.5\%

457 and $>1 \%$ of alleles, respectively and double mutations S436A,A437G and A437G,A581G were

458 observed in $1.1 \%$ and $>1 \%$ of alleles respectively and triple mutations S436A,A437G,K540E was

459 observed in $>1 \%$ of isolates. This suggests separate trajectories to triple mutants

460 S436A,A437G,K540E and A437G,K540E,A581G. The main trajectory to S436A,A437G,K540E 
461 occurs via pathway A437G/K540E/S436A with a second less likely pathway

462 A437G/S436A/K540E. The main trajectory to A437G,K540E,A581G occurs via pathway

463 A437G/K540E/A581G.

464 In Papua New Guinea, most isolates (56.3\%) were the wild-type allele. The most common

465 mutations were single mutation A437G and double mutation A437G,K540E, which were observed

466 in $5.9 \%$ and $29.4 \%$ of isolates, respectively. All other combinations of the four mutations were

467 absent from the isolates. This suggests the trajectory to the double mutation follows the pathway

468 A437G/K540E.

\section{Predicting the most likely evolutionary pathways to resistant mutations in PVDHPS}

470 Homologous mutations in PVDHPS to those in PfDHPS have been reported that confer resistance

471 to sulfadoxine, such as S382F/A/C, A383G, K512E/M/T and A553G, which correspond to

472 PfDHPS mutations S436F/A, A437G, K540E and A581G respectively [16, 49]. It has been

473 suggested that these homologous mutations, most notably A383G and A553G, confer

474 sulfadoxine resistance in P. vivax [50]. Pornthanakasem et al. (2016) [51] reported 30-fold

475 decrease in sulfadoxine binding affinity upon single mutation A383G relative to the wild-type

476 allele, and a further almost 4-fold decrease for the double mutation A383G,A553G relative to

477 A383G, and an almost 5-fold decrease for the triple mutation S328A,A383G,A553G relative to

478 A383G.

479 The single mutation A383G, along with double A383G,A553G and triple mutant

480 S328A,A383G,A553G have been observed in $90 \%$ of mutations in areas where malaria is

481 endemic in Thailand [52]. It is also thought that, like A437G, A383G is likely to be the initial

482 mutation and may be necessary for the appearance of subsequent mutations, as it is most often

483 found in field isolates [49, 52]. In fact, A383G is now so frequent, it its considered fixed in the

484 population, much like its homologous mutation A437G. In our isolate data, the wild-type allele

485 contains a Glycine at position 383, however to test our method we chose Ala-383 as the wild-type

486 residue, and simulated evolutionary trajectories including the A383G mutation. 
487 In our isolate data, mutation Met-205 to lle (M205I) occurs frequently in combination with A383G,

488 A553G and S382A/C. M205I has been previously identified by other studies as a common

489 polymorphism occurring $P$. vivax $[49,51]$, however it is unknown if this mutation confers

490 resistance to sulfadoxine as it occurs in the PPPK gene of the bifunction enzyme PPPK-DHPS. It

491 may be possible, however, that M205I interacts via long-range epistasis with the three other

492 aforementioned PVDHPS sulfadoxine resistance mutations and so we chose to predict the most

493 likely evolutionary pathways to quadruple mutant M205I,S382A,A383G,A553G which was

494 observed at high frequency in our isolate data

495 We generated Flex ddG predictions of the change in sulfadoxine binding free energy for the

496 combinatorically complete set of the four aforementioned mutations (M205I, S382A, A383G,

497 A553G) and also the single mutation V585A. We then compared the peak of the predicted

498 distributions for single mutations A383G and V585A, double mutation A383G,A553G and triple

499 mutation S328A,A383G,A553G to the data presented in [51], and observed a Pearson's

500 correlation of 0.94 with $3 / 4$ of the mutations correctly classified as stabilizing or destabilizing (Table

501 3). Despite the high correlation, the predicted destabilizing effect on sulfadoxine binding free

502 energy for the triple mutation S382A,A383G,A553G was less than both single mutation A383G

503 and double mutation A383G,A553G. This is at odds with the data, where the triple mutation is the

504 most destabilizing mutation of the set studied.

505 Comparing Flex ddG predictions, A383G was the most destabilizing single mutation compared to

506 M205I, S382A and A553G, and was predicted to be 7-times more destabilizing than the second

507 most destabilizing mutation, A553G. Double mutation A383G,A553G was the most destabilizing

508 double mutation and triple mutation M205I,S382A,A553G was the most destabilizing triple

509 mutation.

510 We determined the non-additivity between the impact of multiple mutations and the sum of their

511 independent impacts to investigate epistasis between the mutations (see Supplementary data,

512 'PvDHPS_epistasis.xlsx'). Only one mutation, M205I,A553G appears to be additive, whilst all 
513 other multiple mutations were predicted to be non-additive, suggesting epistasis. All multiple

514 mutations except double mutation S382A,A553G, triple mutation S382A,A383G,A553G and

515 quadruple mutation M205I,S382A,A383G,A553G were more destabilizing than expected. The

516 largest non-additive interactions were for mutations M205I,S382A,A553G and

517 M205I,S382A,A383G,A553G, where M205I,S382A,A553G was predicted to be 9-times more

518 destabilizing than expected, and M205I,S382A,A383A,A553G was predicted to be almost 10-

519 times less destabilizing than expected. Interestingly, quadruple mutation

520 M205I,S382A,A383G,A553G, was predicted to be less destabilizing than single mutation A383G

521 alone.

522 Single mutation M205I occurs in the PPPK gene and is not in direct contact with sulfadoxine and 523 on its own Flex ddG predicts it to have a mostly neutral impact on sulfadoxine binding affinity

$524\left(\Delta \Delta G_{M 205 I}^{*}=-0.03(\right.$ R.E.U)). However, it is predicted to interact epistatically with all mutations,

525 except A553G and A383G,A553G although there are no direct interactions between M205I and

526 the other mutations, suggesting long-range epistasis.

527 We simulated possible evolutionary trajectories to the quadruple mutation

528 M205I,S382A,A383G,A553G (Figure 7a). The most likely first step in the pathway is single

529 mutation A383G, in agreement with the observation that $A 383 G$ is now a major allele. The two

530 most likely pathways to the quadruple mutation are A383G/M205I/S382A/A553G and

531 A383G/M205I/A553G/S382A respectively.

532 Analyzing the frequency of each mutation in our isolate data (Figure 7b), M205I is the most

533 frequent single mutation and $A 383 G$ is the second most frequent, whereas $A 383 G$ and $A 553 G$

534 are the two most likely single mutations in our simulations (Supplementary data

535 'PvDHPS_pathway_probabilities.csv'). The most frequent double mutations in the isolate data

536 and the most likely double mutations in our evolutionary simulations were M205I,A383G and

537 A383G,A553G, when summing over all possible pathways. The most frequent triple mutation in

538 the isolate data, M205I,A383G,A553G, was the second most likely triple mutation in our 
539 simulations, considering all pathways. The most likely triple mutation in our simulations,

540 M205I,S382A,A383G, corresponds to the second most frequent triple mutation in the isolate data.

541 To compare our simulations to the geographical distribution of mutations, we grouped the data

542 into five rough regions: South America (Brazil, Colombia, Guyana, Mexico, Panama, Peru), East

543 Africa (Ethiopia, Eritrea, Madagascar, Sudan, Uganda), South Asia (Afghanistan, Bangladesh,

544 India, Pakistan, Sri Lanka), Southeast Asia (Cambodia, China, Laos, Malaysia, Myanmar,

545 Philippines, Thailand and Vietnam) and Melanesia (Papua New Guinea).

546 If we consider the geographical distribution of PVDHPS mutations (Figure 8), we can see that the 547 dominant mutations differ between regions. In Southeast Asia, many combinations of the set of

548 four mutations M205I, S382A, A383G and A553G are observed. Single mutations M205I, A383G

549 and $A 553 G$ were and observed in $1.2 \%,>1 \%$ and $5.9 \%$ of isolates, respectively. Double

550 mutations M205I,A383G, S382A,A383G and A383G,A553G were observed in $28.2 \%,>1 \%$ and

$551>1 \%$ of isolates, respectively. Triple mutations M205I,S382A,A383G, M205I,A383G,A553G and

552 S382A,A383G,A553G were observed in 1.2\%, 26.1\% and $>1 \%$, respectively, and the quadruple

553 mutation was observed in $11.3 \%$ of isolates. This suggests the evolutionary trajectory to the

554 quadruple mutation occurs main pathways M205I/A383G/A553G/S382A, with possible alternative 555 pathway A383G/M205I/A553G/S382A.

556 However, only a small number of the possible combinations of the four mutations were observed

557 in South Asia, South America, East Africa and Papua New Guinea. In South Asia, mutations

558 A383G, A553G and A383G,A553G were observed in $2.6 \%, 1.8 \%$ and $12.3 \%$ of isolates,

559 respectively. In South America, mutations A383G, M205I and M205I,A383G were observed in

$5606.5 \%, 15.5 \%$ and 26.4\%, respectively. In East Africa, mutations A383G and M205I were

561 observed in $3.6 \%$ and $5.9 \%$ of isolates, respectively, and in Papua New Guinea, single mutation

562 M205I was observed in $15.4 \%$ of isolates.

563 Other frequent multiple mutations include M205I,S382C,A383G which was found in $11.2 \%$ of 564 South America isolates, E142G,M205I and E142G,M205I,A383G,A647V which were found in 
$56536.9 \%$ and $21.4 \%$ of East African isolates, respectively and E132G,A383G,A553G which was

566 found in $8.9 \%$ of Southeast Asian isolates.

567

568

569

570

571

572

573

574

575

576

577

578

579

580

581

582

583

584

585

586

587

588

589

590

\section{Discussion}

We have presented a method for predicting the most likely evolutionary trajectories to multiple mutants, utilizing Rosetta Flex ddG protocol and a thermodynamic evolutionary model. The most likely pathways predicted by our model to the pyrimethamine-resistant quadruple PfDHFR mutant correspond well to those predicted by Lozovsky et al. (2009) [7]. They used experimentally determined IC50 values of PfDHFR pyrimethamine binding for the combinatorically complete set of the four PfDHFR mutations (N51I, C59R, S108N, I164L), combined with information regarding the nucleotide bias of the $P$. falciparum genome, to simulate the evolutionary trajectories. The three most likely pathways based on experimental IC50 values were found in the top four most likely pathways based on our simulations using predictions of binding free energy.

We also simulated the most likely evolutionary trajectories to the PVDHFR quadruple mutation N50I,S58R,S117N,I173L and compared our results to those of Jiang et al. (2013) [8]. They considered the relative growth rates of the different alleles at different drug concentrations when simulating evolutionary trajectories, which incorporate both change in pyrimethamine binding affinity $\left(K_{i}\right)$ and catalytic activity $\left(k_{c a t}\right)$. Our top two most likely pathways correspond to their top two most likely pathways for the highest pyrimethamine concentration they consider, albeit in reverse order. At high pyrimethamine concentrations, it is likely alleles which significantly reduce binding affinity will be selectively favoured even if there is a slight reduction in catalytic activity. This may be why our predictions agree well their predictions for high pyrimethamine concentration, but not for low-to-middle pyrimethamine concentrations, because even though ligand concentration is included in our equation for protein fitness (Eq. 1), our model cannot account for adaptive conflict between $K_{i}$ and $k_{\text {cat }}$. 
591 This highlights a limitation of our method as it only accounts for changes in binding affinity and

592 does not account for changes in protein function. As previously mentioned, DHFR catalyzes the

593 reduction of substrate DHF via oxidation of cofactor NADPH. Therefore, in the case of the DHFR

594 enzyme, a future iteration of the model could include the impact resistance mutations have on

595 binding of these two ligands, as a proxy for changes to enzyme function. However, this would

596 require a much more complex model of protein fitness and would be much more computationally

597 expensive.

598 Mutations occurring at a drug-binding site may also reduce the protein's thermodynamic stability

599 [53] and therefore may not be selected for, even if they improve the resistance phenotype.

600 Therefore, our model may also be improved by including selection for mutations that do not

601 reduce thermodynamic stability relative to the wild-type enzyme. There are several computational

602 methods to predict changes in protein stability upon mutation, including mCSM [54], Rosetta

603 Cartesian ddG [55] and FoldX [56]. However, it must also be noted that most proteins are

604 marginally stable [57-59], a property which may have evolved either as an evolutionary spandrel

$605[60,61]$ (a characteristic that arises as a result of non-adaptive processes which is then used for

606 adaptive purposes [62]) or due to selection for increased flexibility to improve certain

607 functionalities $[63,64]$. Therefore, the model would also have to account for the fact that a

608 resistance mutation that increases protein stability relative to the wild-type stability may also

609 result in a reduction in fitness.

610 The quadruple PVDHFR mutation (N50I,S58R,S117N,I173L) has not been noted in the literature

611 or in our clinical isolate data. This may be because $P$. vivax is only exposed to pyrimethamine

612 when present in co-infections with $P$. falciparum and so is not under continued selection for

613 pyrimethamine resistance. Furthermore, the quadruple mutation only reached fixation in the

614 evolutionary simulations described in [8] for the highest pyrimethamine concentrations, therefore

615 clinical dosages of pyrimethamine may not be high enough to select for the quadruple mutation. 
616 Additionally, the quadruple mutation may result in a fitness impairment that requires

617 compensatory mutations.

618 Despite its limitations, the evolutionary trajectories predicted using our method agree well with

619 experimentally predicted trajectories for both PfDHFR and PVDHFR, and so we applied our

620 method to predict the evolutionary trajectories to quadruple mutants in both PfDHPS and

621 PVDHPS that may confer resistance to sulfadoxine. Evolutionary trajectories to multiple

622 resistance mutations in these enzymes have not yet been investigated fully, either experimentally

623 or computationally, although attempts have been made to infer the first step in sulfadoxine

624 resistance. Therefore, our work presents the first attempt to predict evolutionary trajectories in

625 these enzymes.

626

627 As we can see from the geographical distributions of mutations in PfDHPS (Figure 6) and

628 PVDHPS (Figure 8), there is much more variation between different regions than was observed

629 for the DHFR gene from both species (Figure 2 and Figure 4 for PfDHFR and PVDHFR,

630 respectively). For both species, the quadruple mutations to which we simulated evolutionary

631 trajectories are only observed in Southeast Asia, a region of high drug pressure Furthermore,

632 whilst we have only considered the trajectories for one multiple mutant for each gene, it may be

633 beneficial to consider one per region for the DHPS gene from both species.

634

635 Oguike et al. (2016) [65] reported the emergence of a further PfDHPS sulfadoxine resistance

636 mutation lle-431 to Val (1431V) in Nigeria and found it occurred most commonly as a quintuple

637 mutant with S436A, A437G, A581G and A613S. This quintuple mutation is also found frequently

638 in our isolate data from West Africa, along with single, double, triple and quadruple combinations

639 of the mutations involved. Therefore, it may also be useful to study the evolutionary trajectories to

640 this quintuple mutation. 
641 We might also consider quadruple PvDHPS mutation E142G,M205I,A383G,A647V, which is

642 highly prevalent in East Africa. However, the mutations at codons 142 and 205 occur in the

643 PVPPPK gene of the bifunctional enzyme PPPK-DHPS and are not directly involved in

644 sulfadoxine binding. Therefore, this mutation may not result in a resistant phenotype.

645

646 We also inferred evolutionary pathways from our clinical isolate data from different geographical

647 regions for each drug-target combination studied here. This analysis suggested that different

648 regions often follow different evolutionary trajectories and that the most likely evolutionary

649 trajectories predicted by our model are not always the most prevalent.

650 Geographical differences in the distribution of resistant alleles may be the result of drug regimens

651 and gene flow in parasite populations. If we consider the geographical distribution of PfDHFR

652 mutations (Figure 2), we can see that triple mutant N51I,C59R,S108N is common in West and

653 East Africa as well as Southeast Asia, whereas the quadruple mutation N51I,C59R,S108N,I164L

654 is only observed at high frequency in Southeast Asia. Combination drug SP was first used in

6551967 to treat $P$. falciparum in Southeast Asia, and resistance was first noted that same year on

656 the Thai-Cambodia and Thai-Myanmar borders [66]. In Africa, SP was first used in the 1980s,

657 with resistance occurring later that decade. However, analysis of PfDHFR genotypes and

658 microsatellite haplotypes surrounding the DHFR gene in Southeast Asia and Africa suggest a

659 single resistant lineage that appeared in Southeast Asia accumulated multiple mutations,

660 including the triple N51I,C59R,S108N [67-69], migrated to Africa and spread throughout the

661 continent [70-72]. Variation in the frequency of PfDHFR (and PfDHPS) mutants across Africa

662 occurs because of differences in the timing of chloroquine withdrawal and introduction of SP, as

663 well as continued use of SP for intermittent preventive treatment (IPTp) in pregnant women

664 residing in areas of moderate to high malaria transmission intensity $[48,73]$.

665 Pyrimethamine resistance increased in West Papua in the early 1960s following the introduction

666 of mass drug administration [74]. In our data, the distribution in Papua New Guinea is made up 
667 mainly by the double mutant C59R,S108N and triple mutant C59R,S108N,S306F. Microsatellite

668 haplotype analysis suggests C59R,S108N in Melanesia has two lineages, one of which originated

669 in Southeast Asia whilst the other evolved indigenously [69].

670 Pyrimethamine resistance in South America looks surprisingly different from the distributions in

671 Africa and Southeast Asia. SP was introduced in South America and low-level resistance was first

672 noted in Colombia in 1981 [75]. Microsatellite haplotype analysis suggests pyrimethamine

673 resistance evolved indigenously in South America, with at least two distinct lineages detected. A

674 triple mutant lineage (C50I,N51I,S108N) was identified in Venezuela that possibly evolved from

675 double mutant N51I,S108N [71]. A second triple mutant lineage (N51I,S108N,I164L) was

676 identified in Peru and Bolivia which also possibly evolved from a distinct double mutant

677 (N51I,S108N) lineage [76].

678 The distribution of PVDHFR pyrimethamine-resistance mutations (Figure 4) is much more diverse

679 than the PfDHFR distribution (Figure 2), despite the lower number of PVDHFR isolates. However,

680 the opposite is true for the DHPS gene, with a greater diversity of mutations found the $P$.

681 falciparum isolates.

682 In general, the PVDHFR gene is much more polymorphic than PfDHFR gene, with over 20 alleles

683 observed in a limited geographical sampling [25], whereas fewer PfDHFR alleles have been

684 observed despite much more extensive surveillance with non-synonymous changes and

685 insertions/deletions occurring rarely [77]. It also appears that the origin of PVDHFR

686 pyrimethamine resistance mutation is much more diverse than PfDHFR. Hawkins et al. (2008)

687 [78] investigated isolates from Colombia, India, Indonesia, Papua New Guinea, Sri Lanka,

688 Thailand and Vanuatu. They found multiple origins of the double PVDHFR mutant 58R,117N and

689 three independent origins of triple mutant 58R,61M,117T and quadruple mutants

$69057 \mathrm{I}, 61 \mathrm{M}, 117 \mathrm{~T}, 173 \mathrm{~F}$ and 57L,58R,61M,117T in Thailand, Indonesia and Papua New

691 Guinea/Vanuatu. Shaukat et al. (2021) [79] assessed the evolutionary origin of PVDHFR

692 pyrimethamine resistance mutations in Punjab, Pakistan and found multiple origins of single 
693 mutation S117N and a common origin of double mutant $58 \mathrm{R}, \mathrm{S} 117 \mathrm{~N}$ and triple mutants

$69457 \mathrm{~L}, 58 \mathrm{R}, 117 \mathrm{~N}, 58 \mathrm{R}, 61 \mathrm{M}, 117 \mathrm{~N}$ and $58 \mathrm{R}, 118 \mathrm{~N}, 1173 \mathrm{~L}$. This is in contrast to the evolutionary origin

695 of pyrimethamine resistance in PfDHFR, where mutations in Africa shared a common origin with

696 a resistance lineage from Asia.

697 The distribution of PfDHPS sulfadoxine resistance mutations is strikingly different across different

698 geographical regions (Figure 6). Microsatellite haplotype analysis of resistant alleles revealed

699 single, double and triple sulfadoxine resistance mutations arose independently in Cambodia,

700 Kenya, Cameroon and Venezuela [71, 80]. Microsatellite analysis of PfDHPS in Africa indicated

701 multiple origins of single mutant (A437G) and double mutant (S436A,A437G and A437G,K540E)

702 and different resistance lineages when comparing east and west Africa, resulting in differing

703 distributions of resistance mutations [81].

704 Similarly, multiple origins of PVDHPS resistance mutations at codons 382, 383 and 553 were

705 observed in Cambodia, Indonesia, Sri Lanka and Thailand [49], which may account for the

706 differences in the distribution of mutations between geographical regions (Figure 8).

707 This highlights the need to distinguish between geographical regions and account for existing

708 resistance alleles within that region and trace their lineages when attempting to predict the next

709 step in evolutionary trajectories to highly resistant multiple mutants. Given the current dominant

710 resistance allele from a specific region, our method could be used to predict the most likely next

711 steps from a subset of likely mutations.

712 We have presented a new computational method for predicting the most likely evolutionary

713 trajectories that has demonstrated good agreement with trajectories predicted experimentally and

714 has the advantage of being much quicker and more cost-effective. This method can be applied to

715 any system in which a drug binds to a target molecule, provided a structure of the complex exists

716 or can be produced via structural modelling. Given the threat antimicrobial resistance poses,

717 methods to accurately and efficiently predict future trajectories are vital and can inform treatment

718 strategies and aid drug development. 


\section{Homology modelling}

723 Homology modelling was carried out in Modeller [82] to produce complete structures of the target 724 proteins bound to their drug molecules.

725 Several crystal structures of PfDHFR exist in the Protein Data Bank (PDB). The entry 3QGT

726 provides the crystal structure of wild-type PfDHFR complexed with NADPH, dUMP and

727 pyrimethamine, however residues in the ranges $86-95$ and $232-282$ are missing from the

728 structure. Homology modelling was used to complete the structure using a second wild-type

729 PfDHFR structure PDB entry 1J3I along with a wild-type PVDHFR structure PDB entry 2BLB.

730 To produce a complete structure of PVDHFR, PDB entry 2BLB was used as a template, which

731 provides the X-ray crystal structure of wild-type $P$. vivax DHFR in complex with pyrimethamine.

732 This structure was only missing a loop section between residues 87-105 and so Modeller was

733 used to build this missing loop.

734 To produce a complete structure of PfDHPS, PDB entry 6JWX was used as a template, which

735 provides the crystal structure of wild-type PfDHPS-HPPK in complex with sulfadoxine. This

736 structure is missing a number of loop sections, so Modeller was used to build these loops using

737 additional wild-type PfDHPS template 6JWR and P. vivax HPPK-DHPS template $5 Z 79$.

738 To produce a complete structure of PVDHPS, PDB entry $5 Z 79$ was used as a template, which

739 provides the crystal structure of wild-type $P$. vivax HPPK-DHPS. This structure does not include

740 sulfadoxine, however, and is missing several important loop sections. Modeller was used to build

741 the structure complete with SDX-DHP and completed loops using additional templates 6JWX and

742 6JWA.

$743 \quad$ Flex ddG binding free energy predictions 
744 The Rosetta Flex ddG protocol was used to estimate the change in binding free energy upon

745 mutation, $\Delta \Delta G=\Delta G_{m u t}-\Delta G_{W T}$, for each step in all possible mutational trajectories for a set of

746 stepwise resistance mutations (see supplementary data 'Flex_ddG' folder for example Rosetta

747 script, example resfile and example command line. The protein-ligand structure files and ligand

748 parameter files can be found in the folders named for the specific targets). To predict the change

749 in binding free energy for a single or multiple mutation, we used the structure of the target protein

750 with the drug molecule bound as input to Flex ddG and ran the protocol for 150 times per

751 mutation to produce a distribution of predictions of the change in the free energy of binding. We

752 then found the peak of the distribution to produce a single estimate of the change in the binding

753 free energy for the mutation, denoted $\Delta \Delta G_{X}^{*}$ for mutation $X$.

754 To predict the stepwise evolutionary trajectories, we must consider the interactions between the

755 mutations in the pathway. The interaction energy (or epistasis) in the binding free energy between

756 two mutations $X$ and $Y$, can be written $\varepsilon_{X Y}=\Delta \Delta G_{X, Y}-\left(\Delta \Delta G_{X}+\Delta \Delta G_{Y}\right)$. This quantifies by how

757 much the change in binding free energy of the double mutant $X, Y$ deviates from additivity of the

758 single mutants, where each are calculated with respect to the wild-type. Therefore, the change in

759 binding free energy when mutation $Y$ occurs in the background of mutation $X$ can be written

$760 \Delta \Delta G_{X / Y}=\Delta \Delta G_{X, Y}-\Delta \Delta G_{X}$, where $\Delta \Delta G_{X / Y}=\Delta \Delta G_{Y}+\varepsilon_{X Y}$.

761 For a third mutation, $Z$, occurring in the background of double mutation $X, Y$, the interaction

762 energy between $Z$ and $X, Y$ is $\varepsilon_{X Y, Z}=\Delta \Delta G_{X, Y, Z}-\left(\Delta \Delta G_{X, Y}+\Delta \Delta G_{Z}\right)$. The quantity $\varepsilon_{X Y, Z}$ is not the

763 same as the third order epistasis between mutations $X, Y$, and $Z$, or the interaction energy $\varepsilon_{X Y Z}=$

$764 \Delta \Delta G_{X, Y, Z}-\left(\Delta \Delta G_{X}+\Delta \Delta G_{Y}+\Delta \Delta G_{Z}\right)$, as it does not account for the interaction between $X$ and $Y$,

765 rather it only quantifies the interaction between $Z$ and the two mutations $X$ and $Y$..

766 Therefore, the change in binding free energy when mutation $Z$ occurs in the background of

767 double mutant $X, Y$ can be calculated as $\Delta \Delta G_{X, Y / Z}=\Delta \Delta G_{X, Y, Z}-\Delta \Delta G_{X, Y}$, where $\Delta \Delta G_{X, Y / Z}=\Delta \Delta G_{Z}+$

$768 \varepsilon_{X Y, Z} \cdot$ 
769 To estimate the change in binding free energy when mutation $Y$ occurs in the background of

770 mutation $X, \Delta \Delta G_{X / Y}$ for stepwise pathway $X / Y$, we subtracted the predictions $\Delta \Delta G_{X}^{i}$ for the first

771 mutation $X$, from the predictions for the double mutation $X, Y, \Delta \Delta G_{X, Y}^{i}$, to create a set of 150

772 'predictions' for the change in binding free energy when $Y$ occurs in the background of $X, \Delta \Delta G_{X / Y}^{i}$

773 i.e. $\Delta \Delta G_{X / Y}^{i}=\Delta \Delta G_{X, Y}^{i}-\Delta \Delta G_{X}^{i}$ for $i=\{1, \ldots, 150\}$. To estimate the change in binding free energy

774 when mutation $Z$ occurs in the background of mutations $X$ and $Y$ we calculated. $\Delta \Delta G_{X, Y / Z}^{i}=$

$775 \Delta \Delta G_{X, Y, Z}^{i}-\Delta \Delta G_{X, Y}^{i}$. We applied a similar method for the quadruple mutations, so that we had a set

776 of 'predictions' for each step in the possible evolutionary trajectories.

\section{Simulating Evolutionary Trajectories}

778 To predict the most likely evolutionary trajectories to reach a quadruple mutant we used a model 779 based in thermodynamics and statistical mechanics where the fitness of a protein is determined

780 by the probability it would not be bound to a ligand, $P_{\text {unbound }}$. We consider a two-state system in

781 which the protein can either be bound or unbound and do not explicitly account for if the protein is 782 folded or unfolded in either the bound or unbound state. For ligand concentration $[L]$ it can be 783 shown that the probability a protein is unbound is

$$
P_{\text {unbound }}=\frac{1}{\frac{[L]}{K_{d}}+1}
$$

785 where $K_{d}$ is the protein-ligand dissociation constant and can be calculated as $c_{0} e^{\Delta G / k T}$ where $c_{0}$ is 786 a reference ligand concentration (set here arbitrarily to $0.6 \mathrm{M}$ ), $\Delta G$ is the protein-ligand binding free 787 energy, $k$ is the Boltzmann constant and $T$ is the temperature in Kelvin.

788 Starting from the wild-type protein, with binding free energy $\Delta G_{W T}$ and fitness $P_{\text {unbound }}^{W T}$, we extract 789 one sample $i$ from the 150 values of the predicted binding affinity changes for the single 790 mutations to determine the binding free energy after mutation $X, \Delta G_{X}^{i}=\Delta G_{W T}+\Delta \Delta G_{X}^{i}$, and 
791 calculate the fitness of each single mutant protein $P_{\text {unbound }}^{X(i)}$. We can calculate the probability the 792 mutation will fix in the population using the Kimura fixation probability

$$
p_{f i x}=\frac{1-e^{-2 s}}{1-e^{-4 s N_{e}}}
$$

794 where $N_{e}$ is the effective population size and $s$ is the selection coefficient $s=\left(P_{\text {unbound }}^{X, i}-\right.$

$\left.795 P_{\text {unbound }}^{W T}\right) / P_{\text {unbound }}^{W T}$. We also took in to account the mutational bias of Plasmodium falciparum

796 using the nucleotide mutation matrix calculated in [7]. The probabilities of fixation for each

797 mutation were normalised by the sum of the probabilities of fixation for all possible mutations at

798 that step in the trajectory. A mutation is then chosen with a probability proportional to this

799 normalised probability of fixation.

800 Once a single mutation is chosen, the binding free energy is set to $\Delta G_{X}^{i}$ of the chosen mutation,

801 and a value is sampled from the distribution of each of the possible next steps, $X / Y$ in the

802 trajectory i.e. $\Delta \Delta G_{X / Y}^{i}$. This continues until the end of the trajectory is reached.

\section{SNP data}

Plasmodium falciparum and vivax SNP data were sourced from recent studies [45, 48]. In those studies, paired Illumina raw sequence data was mapped to the Pf3D7 (P. falciparum) or PVP01

806 ( $P$. vivax) reference genome using bwa-mem software (default parameters). SNPs were called 807 using the samtools and GATK software suites. Those SNPs occurring in non-unique, low quality 808 or low coverage regions were discarded, and those in the candidate genes analysed here were extracted.

\section{Acknowledgments}

817

R.C.E and N.F. are funded by the Medical Research Council UK (Grant no. MR/T000171/1). T.G.C is funded by the Medical Research Council UK (Grant no. MR/M01360X/1, MR/N010469/1, MR/R025576/1, and MR/R020973/1) and BBSRC (Grant no. BB/R013063/1). S.C is funded by Medical Research Council UK grants (MR/M01360X/1, MR/R025576/1, and MR/R020973/1) and 
818 Bloomsbury SET. EM is funded by a Newton Institutional Links Grant (British Council, no.

819 261868591. The funders had no role in study design, data collection and analysis, decision to

820 publish, or preparation of the manuscript. R.C.E would like to thank Tanushree Tunstall for her

821 helpful discussions.

822

823

824

825

826

827

\section{References}

828

1. Sun, D., et al., Editorial: Horizontal Gene Transfer Mediated Bacterial Antibiotic

830

831

832

833

Resistance. Frontiers in microbiology, 2019. 10: p. 1933-1933.

2. Davies, J. and D. Davies, Origins and evolution of antibiotic resistance. Microbiology and molecular biology reviews: MMBR, 2010. 74(3): p. 417-433.

3. Levy, S.B. and B. Marshall, Antibacterial resistance worldwide: causes, challenges and responses. Nature Medicine, 2004. 10(12): p. S122-S129.

4. Blair, J.M.A., et al., Molecular mechanisms of antibiotic resistance. Nature Reviews Microbiology, 2015. 13(1): p. 42-51.

5. Weinreich, D.M., et al., Darwinian Evolution Can Follow Only Very Few Mutational Paths to Fitter Proteins. Science, 2006. 312(5770): p. 111.

6. Brown, K.M., et al., Compensatory Mutations Restore Fitness during the Evolution of 7. Lozovsky, E.R., et al., Stepwise acquisition of pyrimethamine resistance in the malaria parasite. Proc Natl Acad Sci, U. S. A., 2009. 106(29): p. 12025-12030.

8. Jiang, P.-P., et al., Accessible Mutational Trajectories for the Evolution of Pyrimethamine Resistance in the Malaria Parasite Plasmodium vivax. Journal of Molecular Evolution, 2013. 77(3): p. 81-91.

9. Sirawaraporn, W., et al., Antifolate-resistant mutants of Plasmodium falciparum dihydrofolate reductase. Proc Natl Acad Sci U S A, 1997. 94: p. 1124-1129.

10. Wellems, T.E., Plasmodium Chloroquine Resistance and the Search for a Replacement Antimalarial Drug. Science, 2002. 298(5591): p. 124-126.

11. Wang, P., et al., Sulfadoxine resistance in the human malaria parasite Plasmodium falciparum is determined by mutations in dihydropteroate synthetase and an additional factor associated with folate utilization. Mol Microbiol., 1997. 23(5): p. 979-986.

12. Organization, W.H., World Malaria Report: 2020. 2020, World Health Organization, Geneva, Switzerland.

13. Brooks, D.R., et al., Sequence variation of the hydroxymethyldihydropterin pyrophosphokinase: dihydropteroate synthase gene in lines of the human malaria parasite, Plasmodium falciparum, with differing resistance to sulfadoxine. Eur, J. Biochem, 1994. 224(2): p. 397-405.

14. Thaithong, S., et al., Pyrimethamine resistant mutations in Plasmodium falciparum. Mol Biochem, Parasitol, 1992. 52(0166-6851 (Print)): p. 149-157.

15. Snounou, G. and N.J. White, The co-existence of Plasmodium: sidelights from falciparum and vivax malaria in Thailand. Trends in Parasitology, 2004. 20(7): p. 333-339.

16. Korsinczky, M., et al., Sulfadoxine resistance in Plasmodium vivax is associated with a specific amino acid in dihydropteroate synthase at the putative sulfadoxine-binding site. Antimicrob Agents, Chemother, 2004. 48(6): p. 2214-2222. 
864 17. Imwong, M., et al., Association of Genetic Mutations inPlasmodium vivax dhfr with Resistance to Sulfadoxine-Pyrimethamine: Geographical and Clinical Correlates. Antimicrobial Agents and Chemotherapy, 2001. 45(11): p. 3122-3127.

18. Leartsakulpanich, U., et al., Molecular characterization of dihydrofolate reductase in relation to antifolate resistance in Plasmodium vivax. Molecular and Biochemical Parasitology, 2002. 119(1): p. 63-73.

19. Hastings, M.D., et al., Dihydrofolate Reductase Mutations in Plasmodium vivax from Indonesia and Therapeutic Response to Sulfadoxine plus Pyrimethamine. The Journal of Infectious Diseases, 2004. 189(4): p. 744-750.

20. Kompis, I.M., R.L. Islam K Fau - Then, and R.L. Then, DNA and RNA synthesis: antifolates. Chem, Rev, 2005. 105(2): p. 593-620.

21. Jiang, T., et al., High prevalence of Pfdhfr-Pfdhps quadruple mutations associated with sulfadoxine-pyrimethamine resistance in Plasmodium falciparum isolates from Bioko Island, Equatorial Guinea. Malar J., 2019. 18(1): p. 101.

22. Berglez, J., et al., Analysis in Escherichia coli of Plasmodium falciparum dihydropteroate synthase (DHPS) alleles implicated in resistance to sulfadoxine. Int, J. Parasitol 2004. 34(1): p. 95-100.

23. Ferlan, J.T., et al., Mutagenesis of dihydrofolate reductase from Plasmodium falciparum: analysis in Saccharomyces cerevisiae of triple mutant alleles resistant to pyrimethamine or WR99210. Mol Biochem, Parasitol, 2001. 113(0166-6851 (Print)): p. 139-150.

24. Yuthavong, Y., et al., Malarial (Plasmodium falciparum) dihydrofolate reductasethymidylate synthase: structural basis for antifolate resistance and development of effective inhibitors. Parasitology,, 2005. 130(0031-1820 (Print)): p. 249-259.

25. Hawkins, V.N., et al., Antifolates can have a role in the treatment of Plasmodium vivax. Trends, Parasitol, 2007. 25(5): p. 213-222.

26. Tamer, Y.T., et al., High-Order Epistasis in Catalytic Power of Dihydrofolate Reductase Gives Rise to a Rugged Fitness Landscape in the Presence of Trimethoprim Selection. Molecular Biology and Evolution, 2019. 36(7): p. 1533-1550.

27. Starr, T.N. and J.W. Thornton, Epistasis in protein evolution. Protein science : a publication of the Protein Society, 2016. 25(7): p. 1204-1218.

28. Khan, A.I., et al., Negative epistasis between beneficial mutations in an evolving bacterial population. Science, 2011. 332(6034): p. 1193-1196.

29. Gong, L.I., M.A. Suchard, and J.D. Bloom, Stability-mediated epistasis constrains the evolution of an influenza protein. eLife, 2013. 2: p. e00631.

30. Sanjuán, R., et al., Epistasis and the adaptability of an RNA virus. Genetics, 2005. 170: p. 1001-1008.

31. Rosenthal, P.J., The interplay between drug resistance and fitness in malaria parasites. Mol, Microbiol, 2013. 89: p. 1025-1038.

32. Melnyk, A.H., A. Wong, and R. Kassen, The fitness costs of antibiotic resistance mutations. Evol, Appl, 2015. 8(3): p. 273-283.

33. Vogwill, T. and R.C. MacLean, The genetic basis of the fitness costs of antimicrobial resistance: a meta-analysis approach. Evolutionary applications, 2015. 8(3): p. 284-295.

34. Costelloe, C., et al., Effect of antibiotic prescribing in primary care on antimicrobial resistance in individual patients: systematic review and meta-analysis. BMJ, 2010. 340 : p. c2096. 
35. Enne, V.I., Reducing antimicrobial resistance in the community by restricting prescribing: can it be done? Journal of Antimicrobial Chemotherapy, 2010. 65(2): p. 179-182.

36. Sundqvist, M., et al., Little evidence for reversibility of trimethoprim resistance after a drastic reduction in trimethoprim use. J. Antimicrob Chemother, 2010. 65: p. 350-360.

37. Andersson, D.I., Persistence of antibiotic resistant bacteria. Curr Opin Microbiol., 2003. 6: p. 452-456.

38. Barlow, K.A., et al., Flex ddG: Rosetta Ensemble-Based Estimation of Changes in ProteinProtein Binding Affinity upon Mutation. The Journal of Physical Chemistry B, 2018. 122(21): p. 5389-5399.

39. Alford, R.A.-O., et al., The Rosetta All-Atom Energy Function for Macromolecular Modeling and Design. J. Chem Theory Comput, 2017. 13: p. 3031-3048.

40. Aldeghi, M., V. Gapsys, and B.L. de Groot, Accurate Estimation of Ligand Binding Affinity Changes upon Protein Mutation. ACS central science, 2018. 4(12): p. 1708-1718.

41. Aldeghi, M., V. Gapsys, and B.L. de Groot, Predicting Kinase Inhibitor Resistance: PhysicsBased and Data-Driven Approaches. ACS Central Science, 2019. 5(8): p. 1468-1474.

42. Eccleston, R.C., D.D. Pollock, and R.A. Goldstein, Selection for cooperativity causes epistasis predominately between native contacts and enables epistasis-based structure reconstruction. Proceedings of the National Academy of Sciences, 2021. 118(16): $p$. e2010057118.

43. Pollock, D.D., G. Thiltgen, and R.A. Goldstein, Amino acid coevolution induces an evolutionary Stokes shift. Proceedings of the National Academy of Sciences, 2012. 109(21): p. E1352.

44. Pollock, D.D., et al., Mechanistic Models of Protein Evolution, in Evolutionary Biology: Self/Nonself Evolution, Species and Complex Traits Evolution, Methods and Concepts, P. Pontarotti, Editor. 2017, Springer International Publishing: Cham. p. 277-296.

45. Benavente, E.D., et al., Distinctive genetic structure and selection patterns in Plasmodium vivax from South Asia and East Africa. Nat Commun, 2021. 12(1): p. 3160.

46. Triglia, T., et al., Mutations in dihydropteroate synthase are responsible for sulfone and sulfonamide resistance in Plasmodium falciparum. Proc Natl Acad Sci, U. S. A., 1997(0027-8424 (Print)): p. 13944-13949.

47. Wang, P., et al., Resistance to antifolates in Plasmodium falciparum monitored by sequence analysis of dihydropteroate synthetase and dihydrofolate reductase alleles in a large number of field samples of diverse origins. Mol Biochem, Parasitol, 1997. 89(01666851 (Print)): p. 161-177.

48. Turkiewicz, A., et al., Genetic diversity of the Plasmodium falciparum GTP-cyclohydrolase 1, dihydrofolate reductase and dihydropteroate synthetase genes reveals new insights into sulfadoxine-pyrimethamine antimalarial drug resistance. PLoS Genet, 2020. 16(12): p. e1009268.

49. Hawkins, V.N., et al., Assessment of the Origins and Spread of Putative ResistanceConferring Mutations in Plasmodium vivax Dihydropteroate Synthase. The American Journal of Tropical Medicine and Hygiene Am J Trop Med Hyg, 2009. 81(2): p. 348-355.

50. Imwong, M., et al., Limited polymorphism in the dihydropteroate synthetase gene (dhps) of Plasmodium vivax isolates from Thailand. Antimicrob Agents Chemother, 2005. 49(10): p. 4393-4395. 


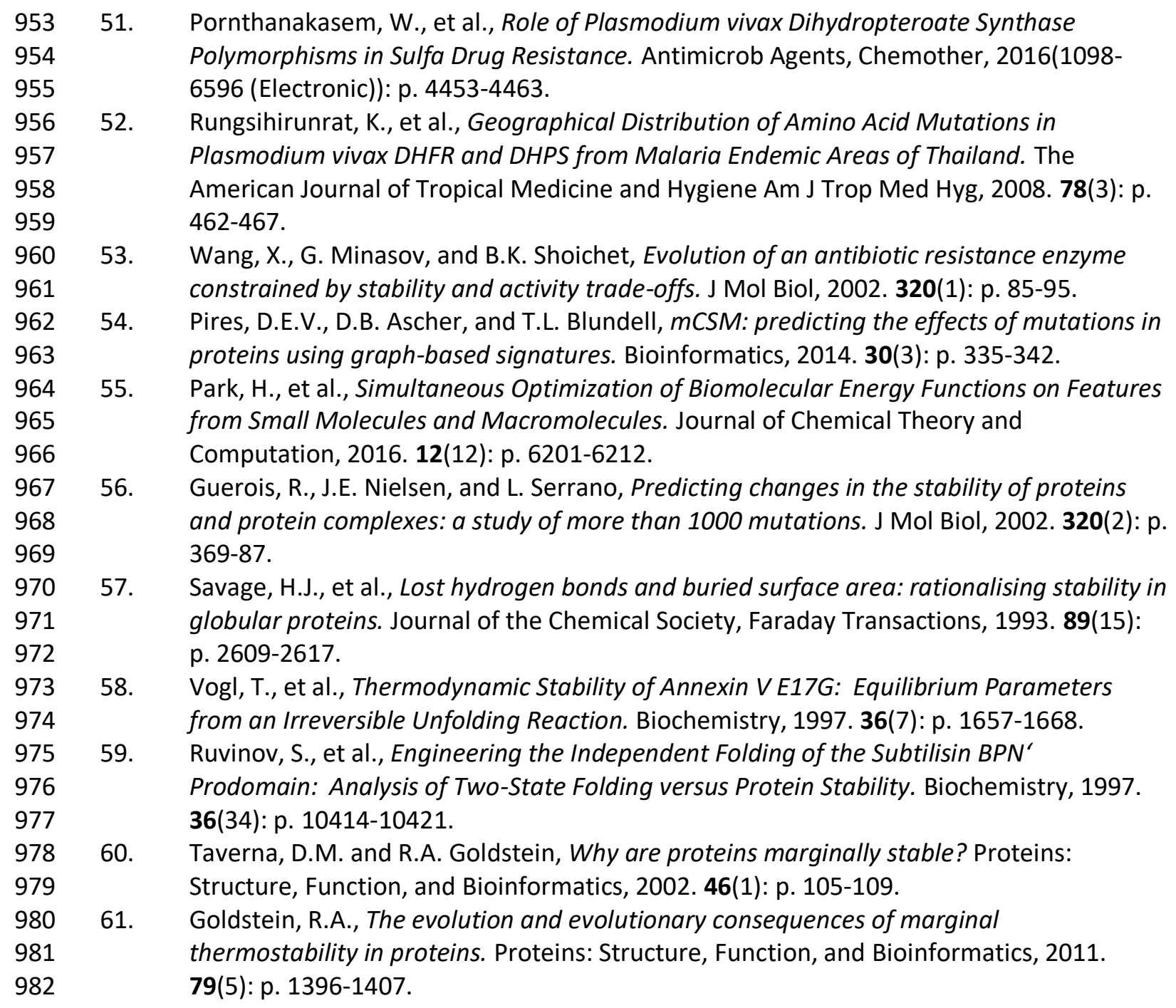

983 62. Gould, S.J., et al., The spandrels of San Marco and the Panglossian paradigm: a critique 984 of the adaptationist programme. Proceedings of the Royal Society of London. Series B. Biological Sciences, 1979. 205(1161): p. 581-598.

\section{Závodszky, P., et al., Adjustment of conformational flexibility is a key event in the} thermal adaptation of proteins. Proceedings of the National Academy of Sciences, 1998. 95(13): p. 7406.

64. Tsou, C.L., Active site flexibility in enzyme catalysis. Ann N Y Acad Sci, 1998. 864: p. 1-8.

65. Oguike, M.C., et al., Molecular determinants of sulfadoxine-pyrimethamine resistance in Plasmodium falciparum in Nigeria and the regional emergence of dhps 431V. Int, J. Parasitol Drugs Drug Resist, 2016.

66. Björkman, A. and P.A. Phillips-Howard, The epidemiology of drug-resistant malaria. Transactions of The Royal Society of Tropical Medicine and Hygiene, 1990. 84(2): p. 177180. malaria parasites. Mol Biol Evol, 2003. 20(9): p. 1526-36. 
998 68. Roper, C., et al., Intercontinental spread of pyrimethamine-resistant malaria. Science,

999

1000

1001

1002

1003

1004

1005

1006

1007

1008

1009

1010

1011

1012

1013

1014

1015

1016

1017

1018

1019

1020

1021

1022

1023

1024

1025

1026

1027

1028

1029

1030

1031

1032

1033

1034

1035 2004. 305(5687): p. 1124.

69. Mita, T., et al., Independent Evolution of Pyrimethamine Resistance in Plasmodium falciparum Isolates in Melanesia. Antimicrobial Agents and Chemotherapy, 2007. 51(3): p. 1071-1077.

70. Maïga, O., et al., A Shared Asian Origin of the Triple-Mutant dhfr Allele in Plasmodium falciparum from Sites across Africa. The Journal of Infectious Diseases, 2007. 196(1): p. 165-172.

71. McCollum Andrea, M., et al., Common Origin and Fixation of Plasmodium falciparum dhfr and dhps Mutations Associated with Sulfadoxine-Pyrimethamine Resistance in a Low-Transmission Area in South America. Antimicrobial Agents and Chemotherapy, 2007. 51(6): p. 2085-2091.

72. McCollum Andrea, M., et al., Hitchhiking and Selective Sweeps of Plasmodium falciparum Sulfadoxine and Pyrimethamine Resistance Alleles in a Population from Central Africa. Antimicrobial Agents and Chemotherapy, 2008. 52(11): p. 4089-4097.

73. Ravenhall, M., et al., Characterizing the impact of sustained sulfadoxine/pyrimethamine use upon the Plasmodium falciparum population in Malawi. Malar J, 2016. 15(1): p. 575.

74. Verdrager, J., Epidemiology of the emergence and spread of drug-resistant falciparum malaria in South-East Asia and Australasia. J Trop Med Hyg, 1986. 89(6): p. 277-89.

75. Espinal, T.C.A., et al., Sensitivity of Plasmodium Falciparum to Antimalarial Drugs in Colombia. The American Journal of Tropical Medicine and Hygiene, 1985. 34(4): p. 675680.

76. Zhou, Z., et al., Decline in sulfadoxine-pyrimethamine-resistant alleles after change in drug policy in the Amazon region of Peru. Antimicrob Agents Chemother, 2008. 52(2): $p$. 739-41.

77. Gregson, A. and C.V. Plowe, Mechanisms of Resistance of Malaria Parasites to Antifolates. Pharmacological Reviews, 2005. 57(1): p. 117.

78. Hawkins, V.N., et al., Multiple origins of resistance-conferring mutations in Plasmodium vivax dihydrofolate reductase. Malaria Journal, 2008. 7(1): p. 72.

79. Shaukat, A., et al., Phylogenetic analysis suggests single and multiple origins of dihydrofolate reductase mutations in Plasmodium vivax. Acta Trop, 2021. 215: p. 105821.

80. Vinayak, S., et al., Origin and Evolution of Sulfadoxine Resistant Plasmodium falciparum. PLOS Pathogens, 2010. 6(3): p. e1000830.

81. Pearce, R.J., et al., Multiple origins and regional dispersal of resistant dhps in African Plasmodium falciparum malaria. PLoS Med, 2009. 6(4): p. e1000055.

82. Webb, B. and A. Sali, Comparative Protein Structure Modeling Using MODELLER. Curr Protoc Bioinformatics, 2016. 54: p. 5.6.1-5.6.37. 
bioRxiv preprint doi: https://doi.org/10.1101/2022.01.25.477595; this version posted February 2, 2022. The copyright holder for this preprint (which was not certified by peer review) is the author/funder, who has granted bioRxiv a license to display the preprint in perpetuity. It is made available under aCC-BY 4.0 International license.

Figures and Tables

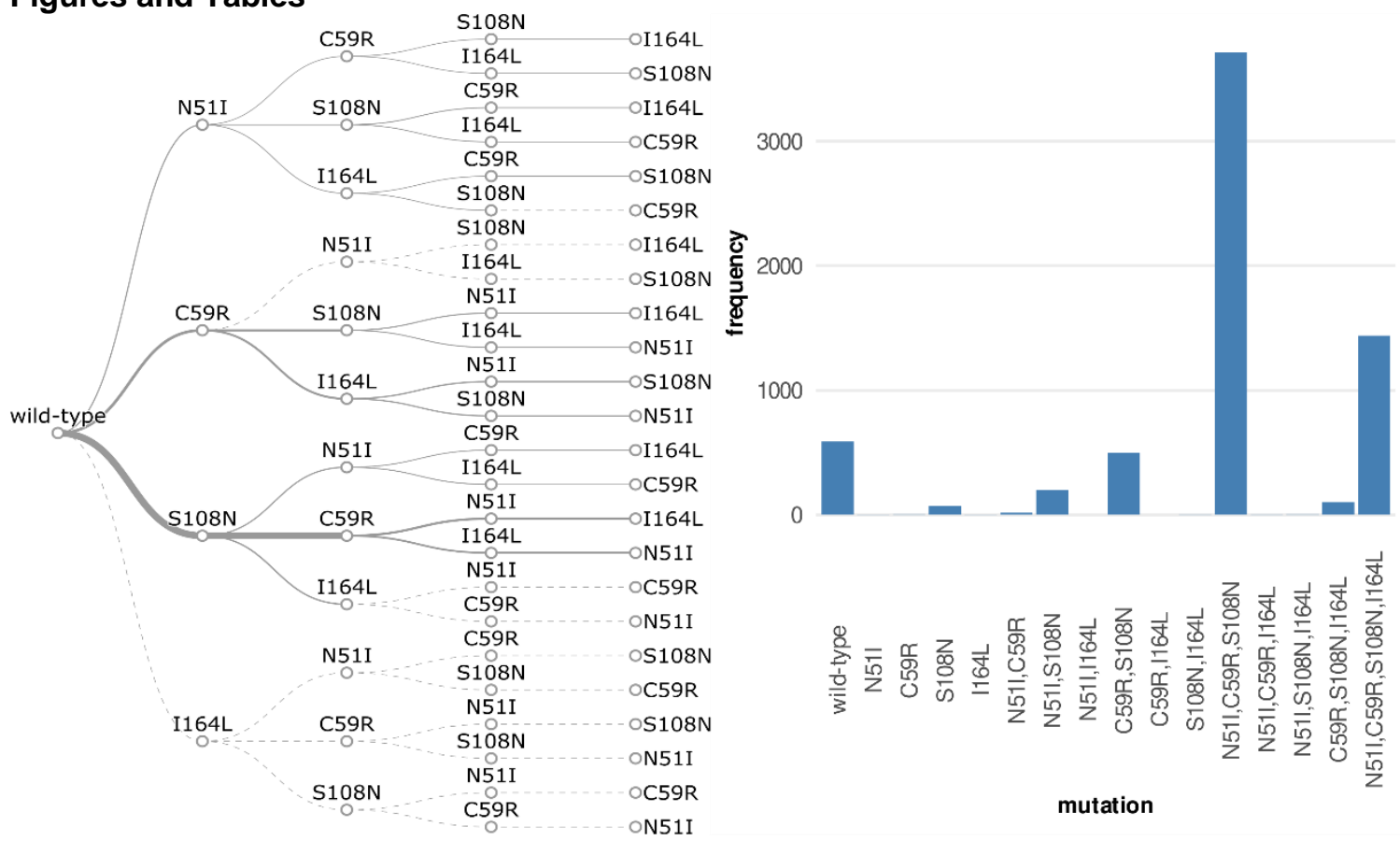

(b)

Figure 1. a) Simulated evolutionary pathways to highly-resistance quadruple PfDHFR mutant N51I,C59R,S108N,I164L. Line thickness indicates the likelihood of a mutation at each step. Dotted lines indicate zero probability of a mutation at that step. The two most likely pathways are S108N/C59R/N51I//164L and S108N/C59R//164L/N51I respectively, b) The frequency of the 16 possible combinations of mutations N51I, C59R, S108N and I164L in PfDHFR, including wildtype, observed in our isolate data. 
bioRxiv preprint doi: https://doi.org/10.1101/2022.01.25.477595; this version posted February 2, 2022. The copyright holder for this preprint (which was not certified by peer review) is the author/funder, who has granted bioRxiv a license to display the preprint in perpetuity. It is made available under aCC-BY 4.0 International license.

Resistance mutations by country

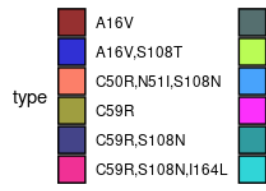
C59R,S108N,S306F C59Y,I164M D $87 \mathrm{H}, \mathrm{S} 108 \mathrm{~N}$ 1164L K256R K256R

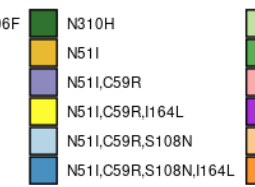

N511,C59R,S108N,N218S N511,C59R,S108N,N461H,D466E,L473I $\square$ S108N N51I,C59R,S108N,S559N N51I,S108N N511,S108N,1164L C59R,S108N,1164L $\square$ M337K

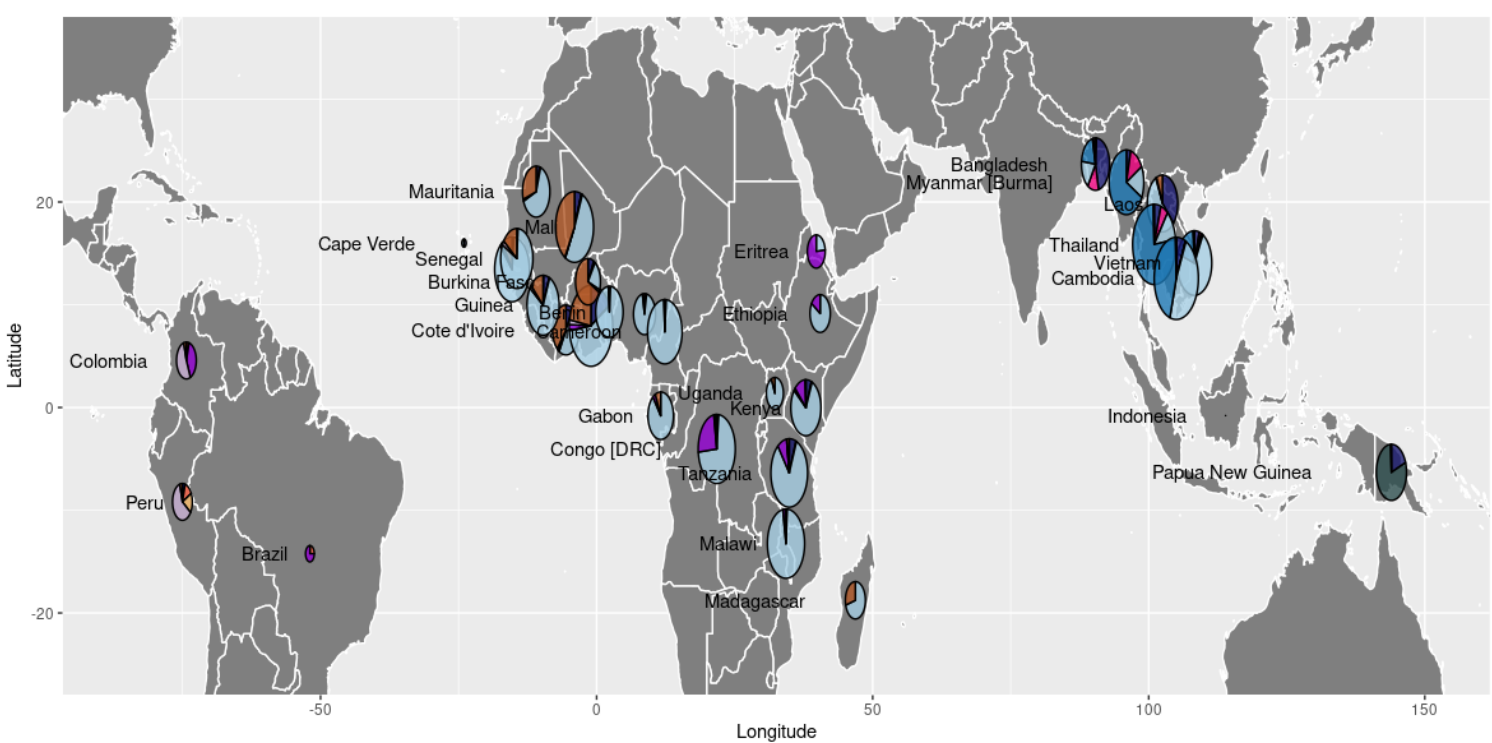

Figure 2. The geographical distribution of mutant PfDHFR alleles found in our isolate data. The size of each pie chart is proportional to the number of isolates from that particular country. 
1071

1072

1073

1074

1075

1076

1077

1078

1079

1080

1081

1082

1083

1084

1085

1086

1087

1088

1089

1090

1091

1092

1093

1094

1095

1096

1097

1098

1099

1100

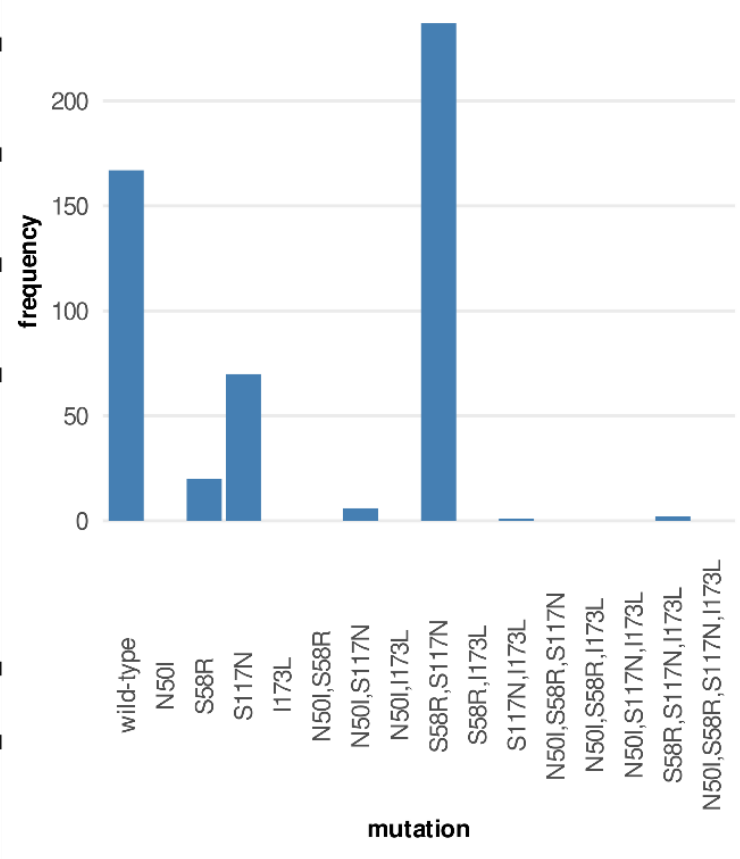

(b)

Figure 3. a) Simulated evolutionary pathways to quadruple PVDHFR mutant N50I,S58R,S117N,I173L. Line thickness indicates the likelihood of a mutation at each step. Dotted lines indicate zero probability of a mutation at that step. The two most likely pathways are S58R/S117N/I173L/N50I and S58R/S117N/N50I/I173L respectively, b) The frequency of the 16 possible combinations of mutations N50I, S58R, S117N and I173L in PVDHFR, including wildtype, observed in our isolate data. 
bioRxiv preprint doi: https://doi.org/10.1101/2022.01.25.477595; this version posted February 2, 2022. The copyright holder for this preprint (which was not certified by peer review) is the author/funder, who has granted bioRxiv a license to display the preprint in perpetuity. It is made available under ACC-BY 4.0 International license.

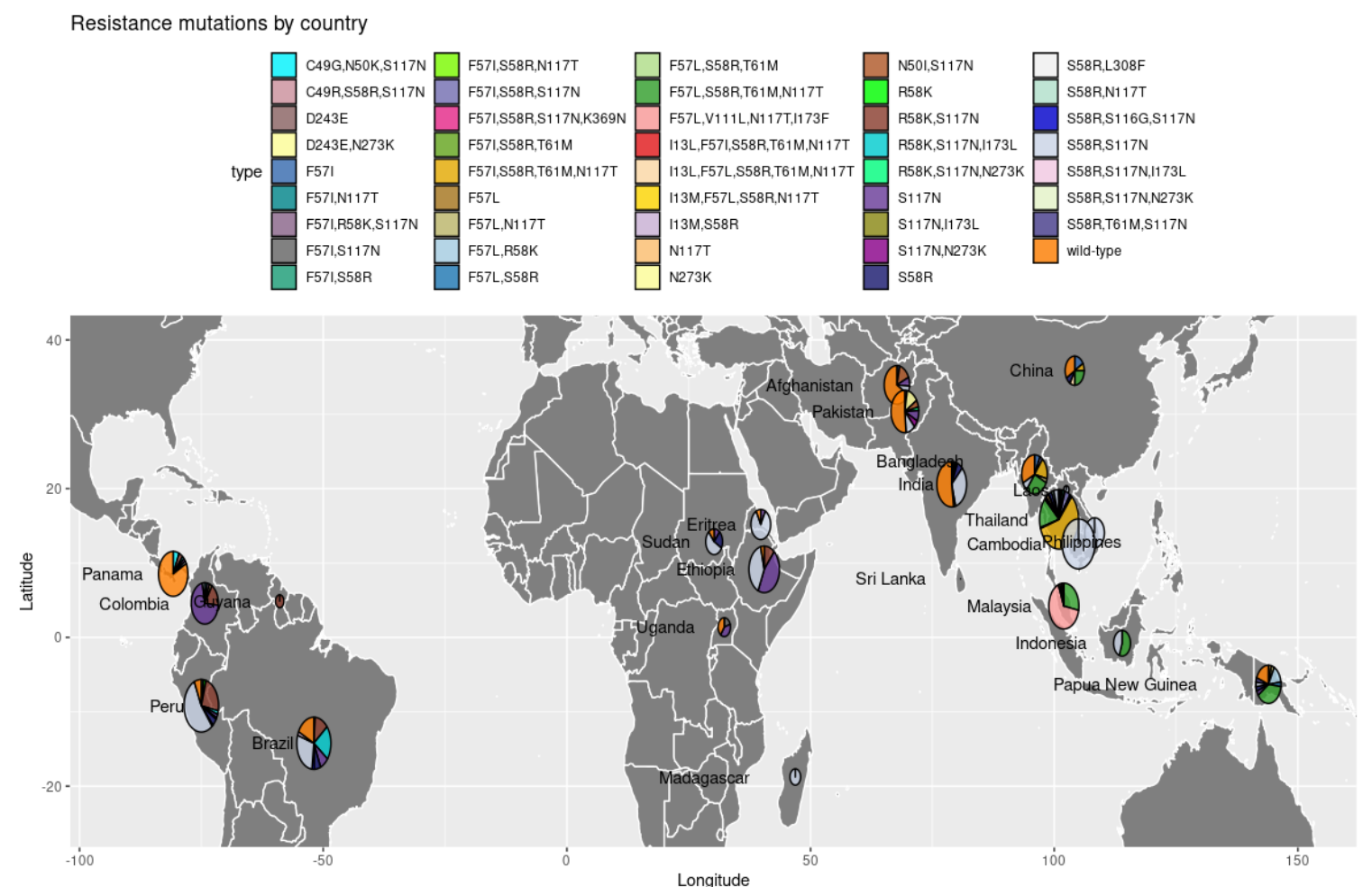

Figure 4. The geographical distribution of mutant PVDHFR alleles found in our isolate data. The size of each pie chart is proportional to the number of isolates from that particular country. 
bioRxiv preprint doi: https://doi.org/10.1101/2022.01.25.477595; this version posted February 2, 2022. The copyright holder for this preprint (which was not certified by peer review) is the author/funder, who has granted bioRxiv a license to display the preprint in perpetuity. It is made available under aCC-BY 4.0 International license.

1126

1127

1128

1129

1130

1131

1132

1133

1134

1135

1136

1137

1138

1139

1140

1141

1142

1143

1144

1145

1146

1147

1148

1149

1150

1151

1152

1153

1154

1155

1156

1157

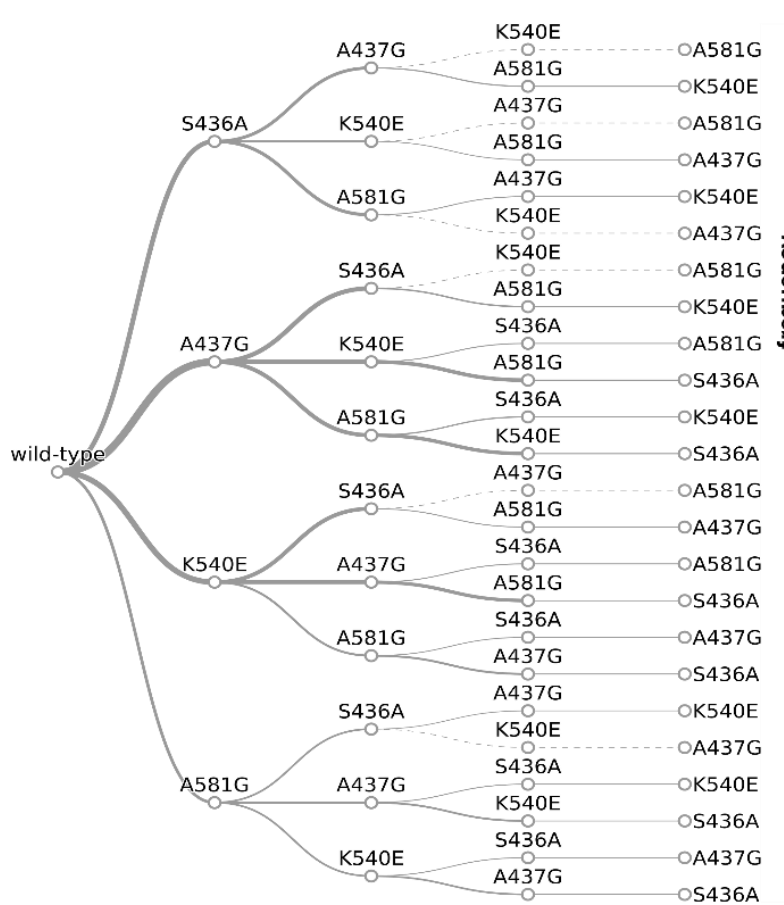

(a)
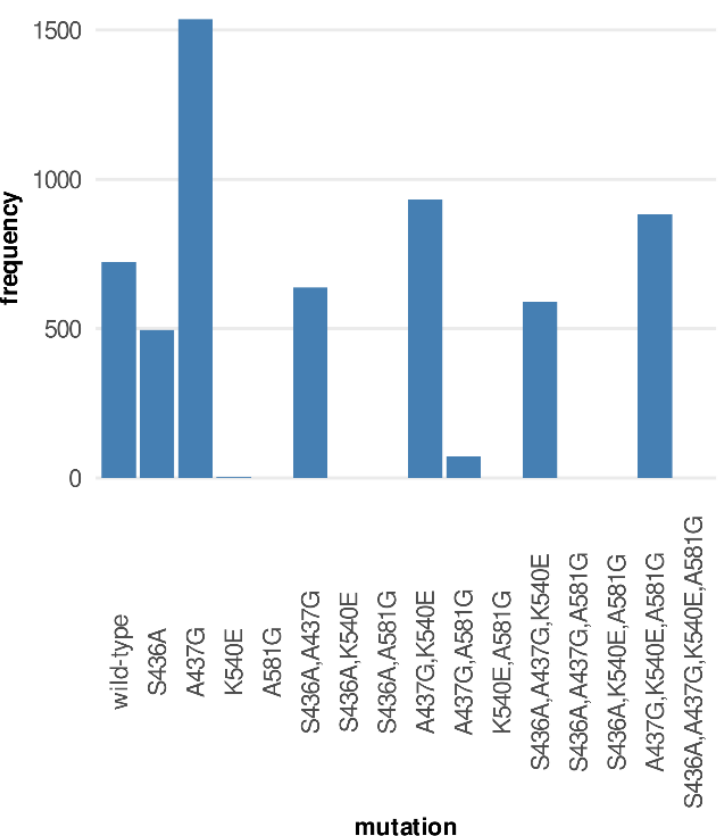

(b)

Figure 5. a) Simulated evolutionary pathways to quadruple PfDHPS mutation

S436A,A437G,K540E,A581G. Line thickness indicates the likelihood of a mutation at each step. Dotted lines indicate zero probability of a mutation at that step. The two most likely pathways are A437G/S436A/A581G/K540E and A437G/K540E/A581G/S436A, b) The frequency of the 16 possible combinations of mutations S436A, A437G, K540E and A581G in PfDHPS, including wild-type, observed in our isolate data. 
bioRxiv preprint doi: https://doi.org/10.1101/2022.01 25.477595; this version posted February 2, 2022. The copyright holder for this preprint (which was not certified by peer review) is the author/funder, who has granted bioRxiv a license to display the preprint in perpetuity. It is made available under aCC-BY 4.0 International license.

Resistance mutations by country

\begin{tabular}{|c|c|c|c|c|c|}
\hline \multirow{12}{*}{ type } & A437G & A437G,K540N,A581G & $1431 \mathrm{~V}, \mathrm{~S} 436 \mathrm{~A}, \mathrm{~A} 437 \mathrm{G}, \mathrm{A} 581 \mathrm{G}, \mathrm{A} 613 \mathrm{~S}$ & S436A,A437G & S436F,A437G \\
\hline & A437G,A581G & A437G,P454T,K540E,A581G & $1431 \mathrm{~V}, \mathrm{~S} 436 \mathrm{~A}, \mathrm{~A} 437 \mathrm{G}, \mathrm{A} 613 \mathrm{~S}$ & $\mathrm{~S} 436 \mathrm{~A}, \mathrm{~A} 437 \mathrm{G}, \mathrm{A} 613 \mathrm{~S}$ & S436F,A437G,A613S \\
\hline & A437G,A613S & A613S & $\mathrm{K} 106 \mathrm{I}, \mathrm{A} 437 \mathrm{G}, \mathrm{K} 540 \mathrm{E}$ & $\mathrm{S} 436 \mathrm{~A}, \mathrm{~A} 437 \mathrm{G}, \mathrm{A} 613 \mathrm{~T}$ & S436F,A437G,A613T \\
\hline & A437G,A613T & C279F & K540E & $\mathrm{S} 436 \mathrm{~A}, \mathrm{~A} 437 \mathrm{G}, \mathrm{K} 540 \mathrm{E}$ & S436F,A437G,K540E,A613S \\
\hline & A437G,D545N & D250V & K540N,A581G & $\mathrm{S} 436 \mathrm{~A}, \mathrm{~A} 437 \mathrm{G}, \mathrm{K} 540 \mathrm{E}, \mathrm{A} 581 \mathrm{G}$ & S436F,A437G,K540E,A613T \\
\hline & A437G,D642N & D278N,A437G & M257K & $\mathrm{S} 436 \mathrm{~A}, \mathrm{~A} 437 \mathrm{G}, \mathrm{K} 540 \mathrm{E}, \mathrm{A} 613 \mathrm{~T}$ & S436F,A613S \\
\hline & A437G,1441M & D280N & M309V & $\mathrm{S} 436 \mathrm{~A}, \mathrm{~A} 437 \mathrm{G}, \mathrm{K} 540 \mathrm{I}$ & $\mathrm{S} 436 \mathrm{P}, \mathrm{S} 436 \mathrm{Y}$ \\
\hline & A437G,1484T & E189Q & N288S,A437G,K540E,A581G & $\mathrm{S} 436 \mathrm{~A}, \mathrm{~A} 437 \mathrm{G}, \mathrm{K} 540 \mathrm{~N}$ & S436P,S436Y,A437G,K540E \\
\hline & A437G,K540E & E189Q,A437G & R243K & $\mathrm{S} 436 \mathrm{~A}, \mathrm{~A} 581 \mathrm{G}, \mathrm{A} 613 \mathrm{~S}$ & $\mathrm{~S} 436 \mathrm{Y}$ \\
\hline & A437G,K540E,A581G & E189Q,S436A & $\mathrm{R} 243 \mathrm{~S}, \mathrm{~S} 436 \mathrm{~A}$ & S436A,A613S & S436Y,A613S \\
\hline & A437G,K540E,V5481,A581G & $1305 \mathrm{~L}$ & S1521,A437G & $\mathrm{s} 436 \mathrm{C}$ & wild-type \\
\hline & A437G,K540N & $1431 \mathrm{~V}, \mathrm{~S} 436 \mathrm{~A}, \mathrm{~A} 437 \mathrm{G}$ & S436A & $\mathrm{S} 436 \mathrm{~F}$ & \\
\hline
\end{tabular}

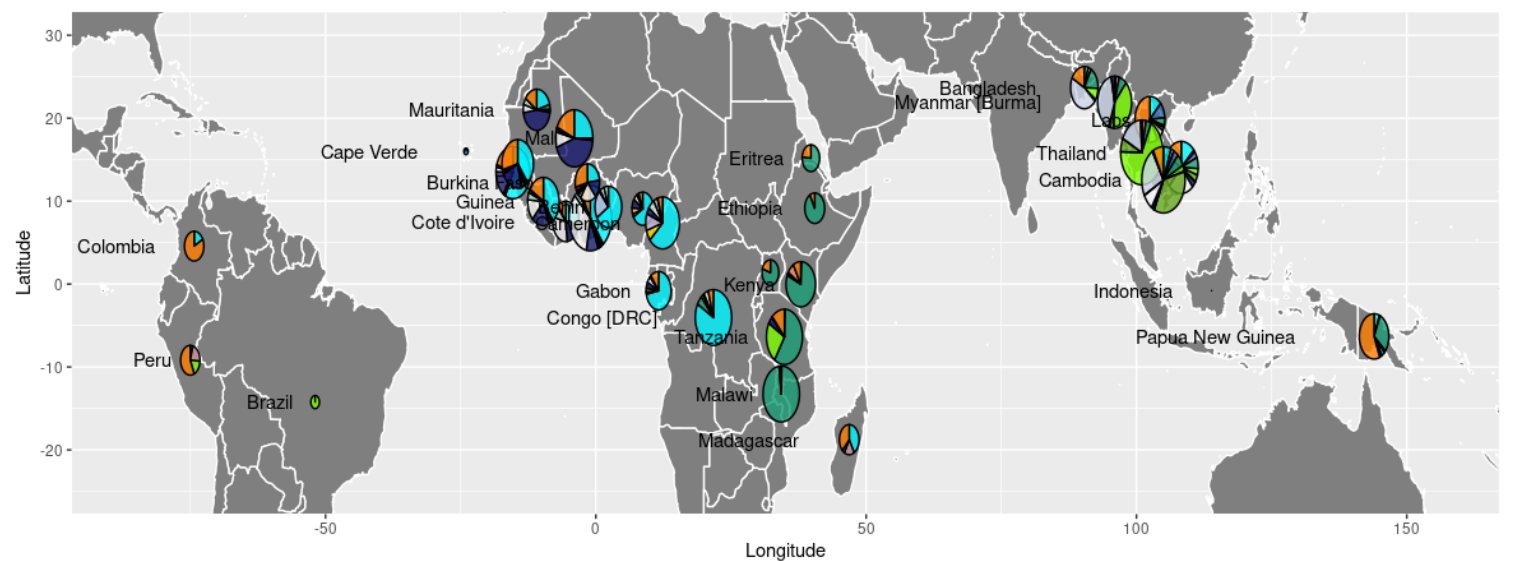

Figure 6. The geographical distribution of mutant PfDHPS alleles found in our isolate data. The size of each pie chart is proportional to the number of isolates from that particular country. 
bioRxiv preprint doi: https://doi.org/10.1101/2022.01.25.477595; this version posted February 2, 2022. The copyright holder for this preprint (which was not certified by peer review) is the author/funder, who has granted bioRxiv a license to display the preprint in perpetuity. It is made available under aCC-BY 4.0 International license.

1179

1180

1181

1182

1183

1184

1185

1186

1187

1188

1189

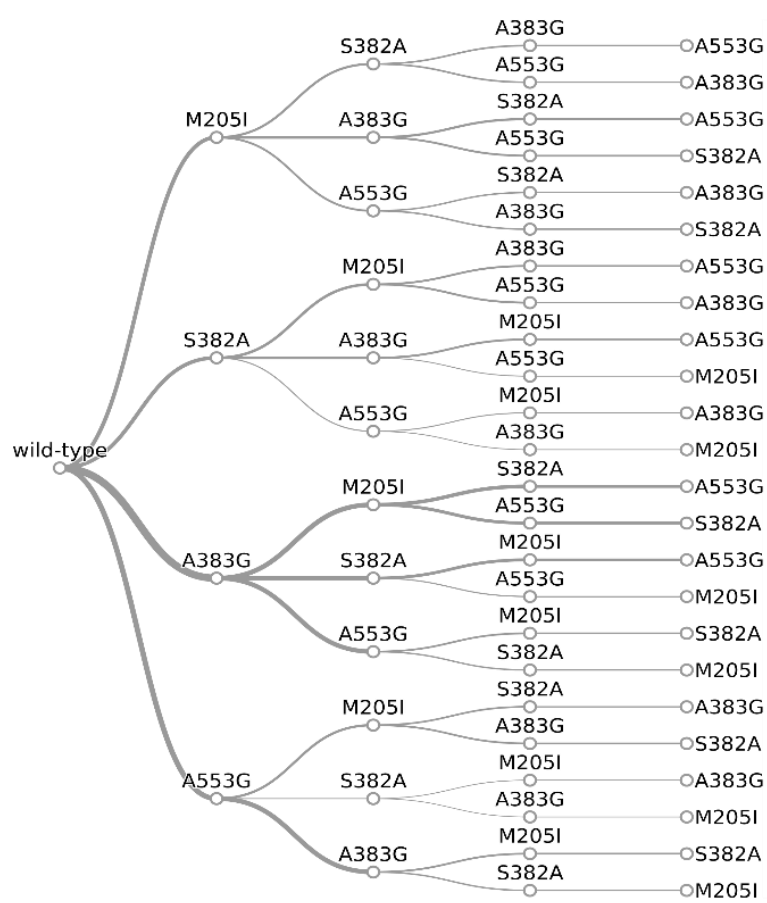

(a)

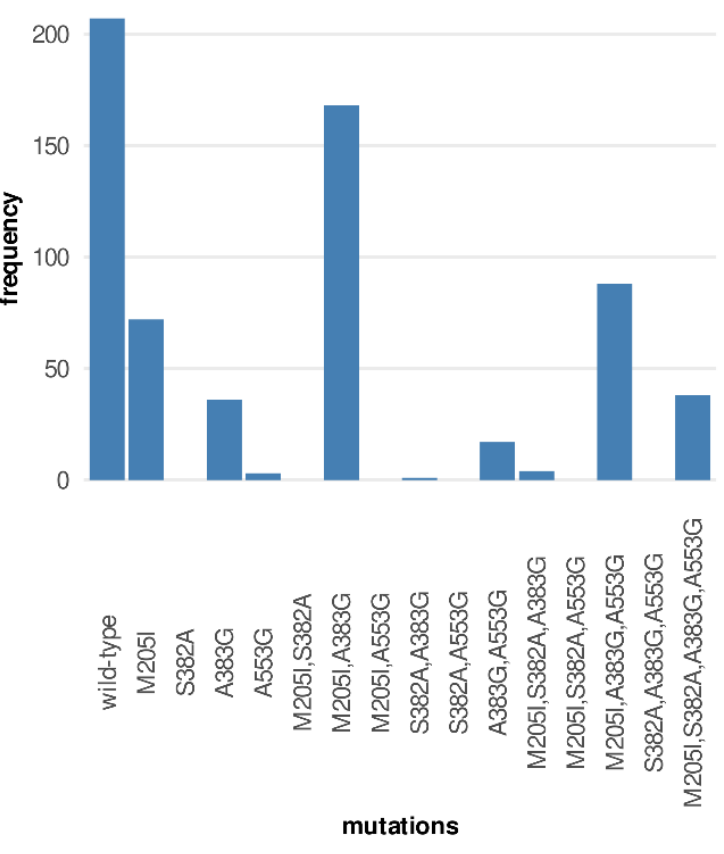

(b)

Figure 7. a) Simulated evolutionary pathways to quadruple PVDHPS mutation M205I,S382A,A383G,A553G. Line thickness indicates the likelihood of a mutation at each step. Dotted lines indicate zero probability of a mutation at that step. The two most likely pathways are A383G/M205I/S382A/A553G and A383G/M205I/A553G/S382A, b) The frequency of the 16 possible combinations of mutations at sites M205I, S382A, A383G and A553G in PVDHPS, including wild-type, observed in our isolate data. 
bioRxiv preprint doi: https://doi.org/10.1101/2022.01.25.477595; this version posted February 2, 2022. The copyright holder for this preprint (which was not certified by peer review) is the author/funder, who has granted bioRxiv a license to display the preprint in perpetuity. It is made available under aCC-BY 4.0 International license.

1190

Figure 8. The geographical distribution of mutant $P V D H P S$ alleles found in our isolate data. The size of each pie chart is proportional to the number of isolates from that particular country.

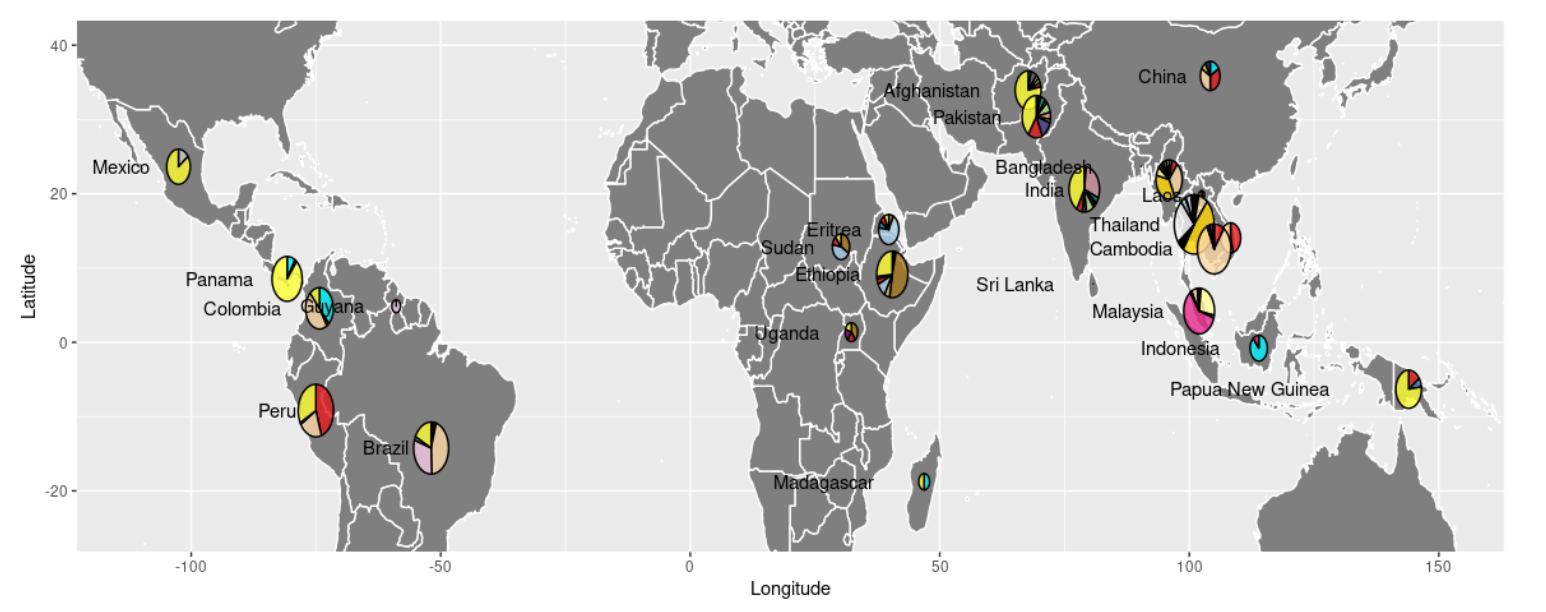
Resistance mutations by country

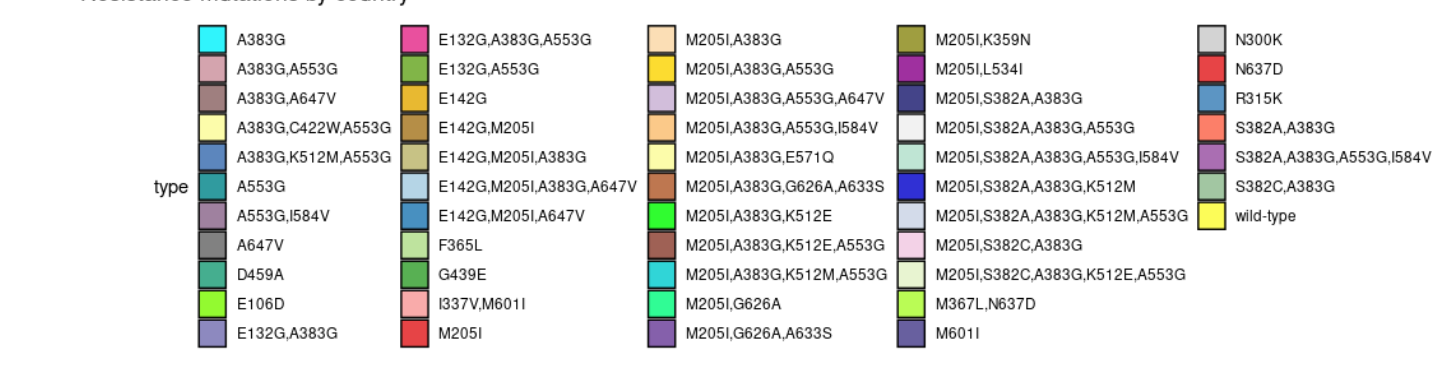


1209 Table 1. Correlation between Flex-ddG predictions and experimental data for PfDHFR 1210 pyrimethamine resistance mutations

1211

1212

1213

1214

1215

1216

1217

1218

1219

1220

1221

1222

1223

1224

1225

1226

1227

1228

1229

1230

1231

1232

1233

1234

1235

1236

1237

1238

1239

1240

1241

1242

1243

1244

1245

1246
Mutation $\Delta \Delta G_{\text {exp }}{ }^{*}(\mathrm{kcal} / \mathrm{mol}) \quad$ Exp. $\quad$ Exp. $\quad \Delta \Delta G_{F l e x d d G}{ }^{\dagger} \quad$ Sum $^{\ddagger} \quad$ I.E. ${ }^{\S}$ Sum $^{\star *}$ I.E. ${ }^{\star * *}$ (R.E.U) $-0.065$ $-0.055$

C59R $-0.184 \quad-0.055$

S108N $1.297 \quad 0.275$

I164L $-0.351 \quad 0.65$

\begin{tabular}{l|llll}
$\mathrm{N} 51 I, S 108 \mathrm{~N}$ & 1.89 & 0.514 & 1.376 & -0.13
\end{tabular}

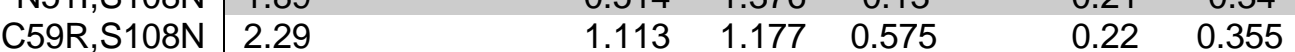

\begin{tabular}{l|llllll} 
N51I,C59R,S108N & 2.595 & 0.33 & 2.265 & 0.225 & 0.155 & 0.07
\end{tabular}

\begin{tabular}{l|llllll} 
C59R,S108N,I164L & 3.283 & 0.762 & 2.521 & 0.025 & -0.43 & 0.455
\end{tabular}

\begin{tabular}{l|llllll} 
N51I,C59R,S108N,I164L & 3.761 & -0.021 & 3.782 & 0.425 & - & 0.92
\end{tabular}

Pearson correlation

$\begin{array}{lll}0.635 & 0.435 & 0.765 \\ 8 / 9 & 4 / 5 & 4 / 5\end{array}$

Correctly classified

*Experimentally measured PfDHFR-pyrimethamine binding free energy change data from [9]

**Sum of experimental values of binding free energy change for independent mutations

***Interaction energy calculated as the difference between experimentally measured values of binding free energy change of multiple mutant compared to the sum of the independent mutations involved.

${ }^{\dagger}$ Change in PfDHFR-pyrimethamine binding free energy predicted by Flex ddG

¥Sum of Flex ddG predictions for independent mutations

§Interaction energy calculated as the difference between Flex ddG predicted binding free energy change of multiple mutant compared to the sum of the independent mutations. 
Table 2. Correlation between Flex ddG predictions and experimental data for PfDHPS

\begin{tabular}{r|ll} 
Mutation & $\Delta \Delta G_{\text {exp }}{ }^{*}(\mathrm{kcal} / \mathrm{mol})$ & $\Delta \Delta G_{\text {Flex ddG }}{ }^{* *}($ R.E.U $)$ \\
\hline A437G & 1.36 & 0.025 \\
A581G & 0.811 & 0.11 \\
S436A,A437G & 2.939 & 0.275 \\
A437G,A581G & 2.817 & 0.125 \\
A437G,K540E & 3.856 & 0.125 \\
Pearson correlation & & 0.469 \\
Correctly classified & & $5 / 5$
\end{tabular}

$1251{ }^{*}$ Change in sulfadoxine binding free energy, calculated from affinity measurements $\left(K_{i}\right)$ from [46] 1252 using the relation $\Delta G=-k T \ln K_{i}$.

$1253{ }^{* *}$ Change in PfDHPS sulfadoxine binding free energy predicted by Flex-ddG 
Table 3. Correlation between Flex ddG predictions and experimental data for PVDHPS sulfadoxine resistance mutations

\begin{tabular}{r|ll} 
Mutation & $\Delta \Delta G_{\text {exp }}{ }^{*}(\mathrm{kcal} / \mathrm{mol})$ & $\Delta \Delta G_{\text {Flex ddG }}{ }^{* *}($ R.E.U) \\
\hline A383G & 2.056 & 1.505 \\
V585A & 0.413 & -0.05 \\
A383G,A553G & 2.826 & 1.875 \\
S382A,A383G,A553G & 3.072 & 1.525 \\
Pearson correlation & & 0.94 \\
Correctly classified & & $4 / 5$
\end{tabular}

$1272 \quad{ }^{*}$ Change in sulfadoxine binding free energy, calculated from affinity measurements $\left(K_{i}\right)$ from [51] 1273 using the relation $\Delta G=-k T \ln K_{i}$.

$1274{ }^{* *}$ Change in PvDHPS sulfadoxine binding free energy predicted by Flex-ddG 